\title{
Opportunistic Routing for Opto-Acoustic Internet of Underwater Things
}

\author{
Abdulkadir Celik, Senior Member, IEEE, Nasir Saeed, Senior Member, IEEE, \\ Basem Shihada, Senior Member, IEEE, Tareq Y. Al-Naffouri, Senior Member, IEEE, \\ and Mohamed-Slim Alouini, Fellow, IEEE
}

\begin{abstract}
Internet of underwater things (IoUT) is a technological revolution that could mark a new era for scientific, industrial, and military underwater applications. To mitigate the hostile underwater channel characteristics, this paper consider a multimodal underwater network that hybridizes acoustic and optical wireless communications to achieve an ubiquitous control and high-speed low-latency networking performance, respectively. Since underwater optical wireless communications (UOWC) suffers from limited range, it requires effective multihop routing solutions. In this regard, we propose a Sector-based Opportunistic Routing (SectOR) protocol. Unlike the traditional unicast routing (TUR) techniques which send packets to a unique relay, opportunistic routing $(\mathrm{OR})$ targets a set of candidate relays by leveraging the broadcast nature of the UOWC channel. OR improves the packet delivery ratio as the likelihood of having at least one successful packet reception is much higher than that in TUR. Contingent upon the performance characterization of a single-hop link, we obtain a variety of local and global metrics to evaluate the fitness of a candidate set (CS) and develop candidate prioritization techniques for various OR metrics. Since rate $\leftrightarrow$ error and range $\leftrightarrow$ beamwidth tradeoffs yield different candidate set diversities, we develop a candidate filtering and searching algorithm to find the optimal sector shaped coverage region by scanning the feasible search space. Moreover, a hybrid acoustic/optic coordination mechanism is considered to avoid duplicate transmission of the relays. Numerical results show that SectOR protocol can perform even better than optimal unicast routing protocols in well-connected underwater networks.
\end{abstract}

Index Terms-Hybrid Underwater Optic Acoustic Networks, Visible Light Communications, Optical Wireless Communications, Opportunistic Routing, Internet of Things.

\section{INTRODUCTION}

$\mathrm{O}$ CEANS cover approximately \%71 of the Earth's surface and provide significant benefits to humanity, such as climate regulation, transportation, food supply, recreation, medicine, and a variety of natural resources [2]. In order to protect oceans and reap their full profits at the same time, it is crucial to transport, store, organize and process the surging amount of data acquired from underwater sensors and fixed/mobile maritime terminals. To this aim, the internet of underwater things (IoUT) is a technological revolution towards integrating physical and digital worlds by interconnecting smart underwater objects [3]. Thus, IoUT could mark a new era for scientific, industrial, and military underwater

Authors are with Computer, Electrical, and Mathematical Sciences and Engineering Division at King Abdullah University of Science and Technology (KAUST), Thuwal, 23955-6900, KSA (Corresponding Author: Abdulkadir Celik). A part of this work was presented in IEEE WCNC 2019 in Marrakesh, Morocco [1]. applications, e.g., disaster prevention, offshore exploration, environmental monitoring, tactical surveillance, and assisted navigation.

Emerging IoUT applications demand an ambitious quality of service, which necessitates high-speed, ultra-reliable, and low latency underwater networking solutions. However, such goals pose daunting challenges for most electromagnetic frequencies due to the hostile channel impediments of the aquatic medium. Although underwater acoustic communication (UAC) is a proven technology that is widely adopted by existing underwater applications, its limited bandwidth and low achievable rates are not sufficient for emerging IoUT applications. Despite its desirable omnidirectional transmission and several kilometers long transmission ranges, the low propagation speed of acoustic waves $(1500 \mathrm{~m} / \mathrm{s})$ yields a high latency that disrupts the proper functioning of long-range applications, especially for real-time operation and synchronization tasks [4]. Moreover, acoustic signals' omnidirectional and longrange propagation nature make UAC systems susceptible to remote eavesdroppers and interceptors.

Underwater optical wireless communication (UOWC) has recently gained attention by its advantages of higher bandwidth, lower latency. Nonetheless, UOWC systems are mainly restrained by their directivity and short communication range, which is primarily driven by the following phenomena [2]: The transmitted light intensity reduces along the propagation path. This energy dissipation is referred to as absorption and is caused by the transformation of photon energy into the internal energy of the absorber. Unlike the ballistic photons, some other photons deflect from the default propagation path; this is also known as scattering and caused either by water particles of a size comparable to the carrier wavelength (i.e., diffraction) or by constituents with different refraction index (i.e., refraction). That is, the relation between absorption and scattering primarily characterizes the fundamental tradeoff between range and beam divergence angle. Nonetheless, the directivity and relatively limited range facilitate the UOWC's innate physical layer security attributes since eavesdropping/interception is only possible for nearby intruders located within the line of sight between the optical transceivers.

Considering the sparse nature of underwater networks, developing multihop communication and routing strategies is of the utmost importance to extend communication ranges and realize underwater optical wireless networks (UOWNs) in real-life. In particular, the design and provision of advanced routing protocols top the list of open networking problems as 
it couples medium access control issues with unique physical layer characteristics of UOWCs. First and foremost, existing routing protocols developed for omnidirectional terrestrial wireless sensor networks and underwater acoustic networks (UANs) cannot be used for UOWNs in a plug-and-play fashion. Due to the directed nature of the light propagation, the coverage region of a light source is in a sector shape whose central angle (i.e., the divergence angle of the light beam) and radius (i.e., communication range) are inversely proportional. Hence, a wide divergence angle allows reaching nearby neighbors, whereas employing a narrow divergence angle renders communicating with a distant node [5]. While the latter requires less number of hops to reach the destination at the cost of equipping the transceivers with accurate pointingacquisitioning-tracking (PAT) mechanisms, the former may operate without PAT at the expense of a higher number of hops and power consumption [6]. Indeed, recent commercial products show that light-emitting diode (LED)-based widebeam UOWC transmitters can reach several ten-meters coverage [7]-[9]. On the other hand, the transmission range of recent narrow-beam laser diode (LD)-based prototypes and commercial products are rated between 100-150 meters [10][12]. Even though these ranges are sufficient for singlehop UOWC applications such as cellular UOWNs and data aggregation via autonomous underwater vehicles [c.f. Fig 1 of [3]], realizing a large-scale UOWN is still a challenging task that requires effective multihop communications and routing methods, which is the main focus of this paper.

Apart from the traditional unicast routing (TUR) protocols that transmit packets to a unique next-hop forwarder, opportunistic routing (OR) broadcasts packets to a set of candidate nodes. TUR commonly protocols retransmit lost packets to the forwarder, which are eventually discarded after a specific number of re-transmission. On the contrary, by leveraging the broadcast nature of UOWC, OR involves other candidates in forwarding the packets if the chosen forwarder fails to receive the packet. For instance, Fig. 1 demonstrates two different routes: The former is the route when the highest priority node (green) successfully receives the packet while the latter route is over the second-highest priority node (red) when the highest priority fails to receive packet correctly. The first case (Route 1) consists of the source node, green node, purple node, orange node, and the sink node, while in the second case (Route 2), the path consists of the source node, red node, yellow node, purple node, and the sink node. Hence, OR improves the packet delivery ratio as the likelihood of having at least one successful packet reception is much higher than that in TUR. Also, OR reduces the expected number of retransmissions, which improves the energy efficiency and delay of the network. Therefore, the proposed OR scheme improves the key performance metrics of the opto-acoustic IoUT network, such as throughput, energy consumption, and delay. Furthermore, OR is especially suitable to UOWNs because of the connection interruptions caused either by underwater channel impediments (e.g., pointing errors, misalignment, turbulence, etc.) or sea creatures passing through the transceivers' lineof-sight. Nonetheless, OR requires effective cooperation and coordination mechanisms among the candidate nodes to avoid

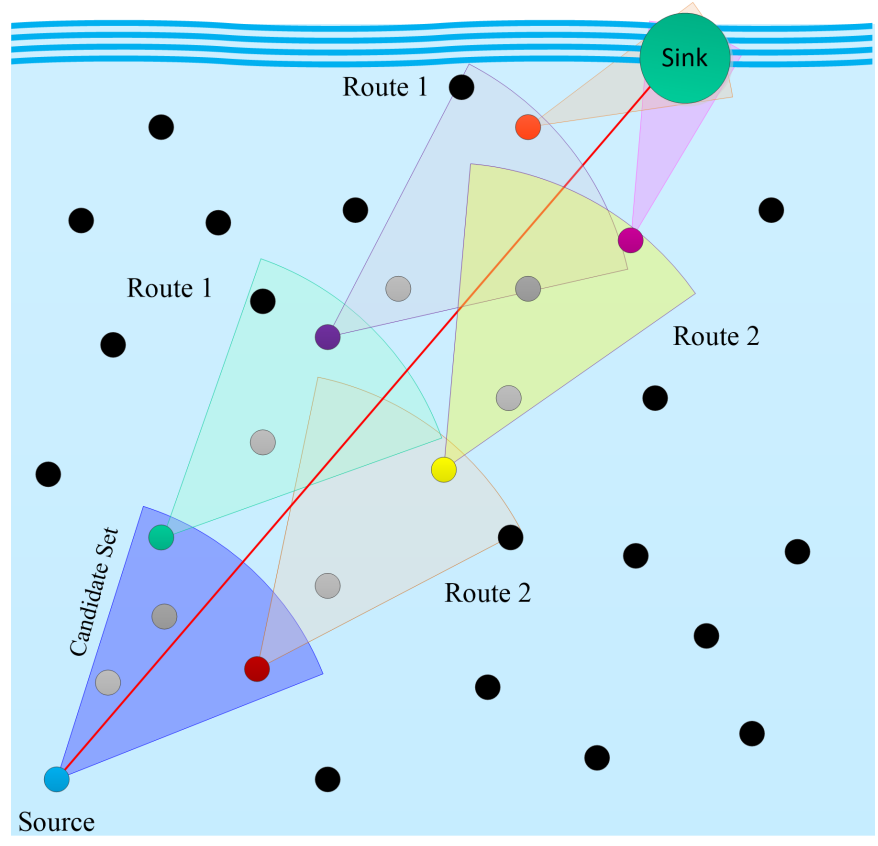

Fig. 1: Illustration of UOAN and SectOR protocol.

duplicate transmissions and collisions.

\section{A. Prior Arts and Main Contributions}

The IoUT concept is surveyed in [13] where authors consider software-defined IoUT nodes that can employ acoustic, optic, and magnetic induction signals to overcome the peculiarities of the underwater environment. A softwaredefined opto-acoustic IoUT network architecture design is also proposed in [3] where authors explain inextricably interwoven relations among functionalities of different layers and introduce network function virtualization (NFV) to realize application specific cross-layer protocol suites through an NFV management and orchestration system.

Although physical layer issues of UOWC is relatively mature, its networking aspects still stays unexplored. Recent efforts on UOWNs can be exemplified as follows: Assuming a Poisson point process based spatial distribution, Saeed et. al. analyzed the $k$-connectivity of UOWNs [14]. In [15], authors characterized the performance of relay-assisted underwater optical CDMA system where multihop communication is realized by chip detect-and-forward method. Similarly, Jamali et. al. consider the performance analysis of multihop UOWC using decode-and-forward (DF) relaying [16]. In [5], we addressed modeling and end-to-end performance analysis of multihop UOWNs under both DF and amplify-and-forward methods. Multihop communication framework presented in [5] is later extended to investigate the impacts of location uncertainty on key performance metrics such as achievable rate, bit error rate, and power consumption [6]. Excluding [5], [6], these works do not deal with the effective UOWN routing protocols which is of utmost importance to extend the limited communication range of UOWCs. In [5], [6], proposed protocols follow a traditional unicast routing approach which adapts shortest path algorithms to find paths 
with minimum distance/error/power consumption or maximum rate. In [17], Diamant et al. propose a routing protocol for multi-modal underwater systems that incorporate optic and acoustic systems to leverage the advantages of both. The proposed routing technique makes distributed decisions on the per-link flow, avoids bottlenecks, and allows simultaneous acoustic and optical transmissions to forward packets towards the destination. Albeit their valuable contributions, the authors do not take the fundamental range-beamwidth tradeoff of UOWC, which has direct and significant impacts on network connectivity and link quality. We should note that above works consider traditional unicast relaying techniques and routing protocols.

Being inspired by the sector-shaped coverage region of the light sources, this paper proposes a Sector-based Opportunistic Routing (SectOR) protocol. The SectOR discovers routing paths in a distributed manner by exploiting local or global OR metrics, respectively. The SectOR is a geographical routing protocol and computes OR metrics by using node location information, which is already required to establish directed links between the nodes. Noting that the OR is extensively studied for terrestrial wireless networks [18, and references therein] and UANs [19]-[22], the SectOR is first to develop an OR protocol for UOWNs to the best of authors' knowledge. The SectOR is significantly different from these OR variants since it tackles fundamental UOWC physical layer tradeoffs to determine candidate sets and select the forwarder nodes. To this aim, the SectOR considers UOWC transmitters with adaptive divergence angles to manipulate range-beamwidth tradeoff, which is well-studied technique in the literature [23]-[27]. The SectOR is also distinct from well-known geographical routing protocols developed for UANs, such as vector-based forwarding (VBF) [28], directional floodingbased routing (DFR) [29], and focused beam routing (FBR) [30]. Since the UACs can reach far away nodes in any direction, these protocols commonly define hypothetical pipeshaped or triangle-shaped routing regions to keep the path between the source and destination. On the other hand, the SectOR dynamically adjusts the sector-shaped UWOC coverage region's size and direction by manipulating the real-life hardware parameters and fundamental tradeoffs. Furthermore, the SectOR is an opto-acoustic (i.e., multimodal) OR scheme, where omnidirectional and long-range acoustic links provide several advantages: 1) acoustic systems can be used to create reliable command and control links. For example, the acoustic links can carry necessary pointing and alignment control data to re-establish broken optical links between disconnected nodes; 2) acoustic links can enable a $360^{\circ}$ neighbor discovery and help sending ACK/NACK messages to candidate set in case of optical link failures; and 3) hybridizing acoustic and optic links improves the localization accuracy [3], [31], which yields precise pointing between the optical transceivers and enhances the end-to-end link quality. The main contributions of this paper can be summarized as follows:

- Based on unicast link performance analysis, the performance of broadcast links are characterized in terms of data rate, maximum range, packet delivery ratio, and expected number of retransmissions. Using these performance characterizations as building blocks, we then developed both local and global OR metrics, such as distance progress, energy efficiency, and low latency.

- Since pointing direction and range $\leftrightarrow$ beamwidth tradeoff yield different candidate set diversities, a candidate filtering and selection technique is proposed to find the best pointing and divergence angle. By manipulating the pointing angles and leveraging adaptive beamwidths, we find the candidate set that delivers the best OR metric of interest. Based on this technique, each node maintains its best pointing and divergence angle, and forward received packets along with the priority order of its candidates. In this way, the SectOR is also applicable for mobile underwater networks. Moreover, neighbor discovery and candidate coordination are facilitated by acoustic communications to mitigate the directivity and range limitations of UOWC.

\section{B. Notations and Paper Organization}

Throughout the paper, sets and their cardinality are denoted with calligraphic and regular uppercase letters (e.g., $|\mathcal{X}|=X$ ), respectively. Vectors and matrices are represented in lowercase and uppercase boldfaces (e.g., $\boldsymbol{x}$ and $\boldsymbol{X}$ ), respectively. Superscripts $s, d, i$, and $j$ are used for indexing source, destination, current forwarder, and next forwarder nodes, respectively. The optimal/best values of variables are always marked with superscript $\star$, e.g., $x_{s d}^{i \star}$.

The remainder of the paper is organized as follows: Section III introduces the network and channel models. Section III analysis the performance of unicast and broadcast links. These performance characterizations are then used to develop local and global OR metrics in Section IV Section V provide the details of the proposed SectOR protocol and summarizes the algorithmic implementation. Section VI presents the numerical results. Finally, Section VII concludes the paper with a few remarks.

\section{UNDERWATER OPTO-ACOUSTIC NETWORKS}

In this section, we introduce the UOAN of interest, present the UOWC channel model, and explain the tradeoff between communication range and beamwidth.

\section{A. Network Model}

We consider a UOAN that consists of a single sink/surface station and M IoUT nodes, as demonstrated in Fig. 1. IoUT nodes are equipped with low-cost optical transceivers to enable UOWC in both forward and backward directions. Light sources are assumed to be capable of adapting their beamwidth and communication range by adjusting the divergence angle [32]. Although optical transceivers are primarily employed to deliver a large volume of sensing data via high-speed UOWC links, the limited range and directivity of UOWC hinder its ability to serve as a reliable control medium for network management tasks. Thanks to its omnidirectional propagation characteristics, each node also has a single acoustic transceiver to 


\begin{tabular}{|c|c|}
\hline \multicolumn{2}{|r|}{ Table of Notations } \\
\hline Not. & Description \\
\hline$n_{i}$ & Node $i$ located at $\ell_{i}, i \in[1, M]$ \\
\hline$n_{s} / n_{d}$ & The ource/destination node. \\
\hline$n_{i} \rightarrow n_{j}$ & The link from $n_{i}$ to $n_{j},(i, j) \in[1, M], i \neq j$ \\
\hline$f_{i}^{j}$ & Photon Arrival rate of $n_{i} \rightarrow n_{j}$ \\
\hline $\mathrm{R}_{\mathrm{i}}^{\mathrm{j}}$ & Data rate of link $n_{i} \rightarrow n_{j}$ \\
\hline $\mathrm{D}_{\mathrm{i}}^{j}$ & Communciation range from $n_{i}$ toward $n_{j}$ \\
\hline $\overrightarrow{B E R_{\mathrm{i}}^{j}}$ & Unicast bit error rate (BER) $n_{i} \rightarrow n_{j}$ \\
\hline$\overline{P E R_{\mathrm{i}}^{j}}$ & Unicast packer error rate (PER) of $n_{i} \rightarrow n_{j}$ \\
\hline $\overrightarrow{P D R_{i}^{j}}$ & Unicast packet delivery ratio (PDR) of $n_{i} \rightarrow n_{j}$ \\
\hline $\mathcal{P}_{i}^{j}(k)$ & Prob. of a delivery over $n_{i} \rightarrow n_{j}$ in $k$ transmissions. \\
\hline $\overrightarrow{N_{i}^{j}}$ & Unicast expected number of transmissions (ExNT) of $n_{i} \rightarrow n_{j}$ \\
\hline $\mathcal{C}_{s d}^{i}$ & Candidate set of $n_{i}$ to forward packets from $n_{s}$ to $n_{d}$ \\
\hline $\mathrm{PER}_{\mathrm{sd}}^{\mathrm{i}}$ & Broadcast PER of $n_{i}$ over CS $\mathcal{C}_{s d}^{i}$ \\
\hline $\mathrm{SFR}_{\mathrm{sd}}^{\mathrm{i}}$ & Broadcast successful forwarding ratio (SFR) of $n_{i}$ over CS $\mathcal{C}_{s d}^{i}$ \\
\hline $\mathcal{P}_{s d}^{i j}(k)$ & Prob. of a delivery over $n_{i} \rightarrow n_{j}$ in $k$ transmissions, $j \in \mathcal{C}_{s d}^{i}$. \\
\hline$\overline{\mathrm{N}}_{\mathrm{sd}}^{\mathrm{i}}$ & Broadcast ExNT of $n_{i}$ over CS $\mathcal{C}_{s d}^{i}$ \\
\hline $\mathrm{DP}_{\mathrm{sd}}^{\mathrm{ij}}$ & Distance progress (DP) metric of $n_{j}, j \in \mathcal{C}_{s d}^{i}$ \\
\hline $\mathrm{EDP}_{\mathrm{sd}}^{\mathrm{ij}}$ & Expected DP (EDP) metric of $n_{j}, j \in \mathcal{C}_{s d}^{i}$ \\
\hline $\mathrm{EEM}_{\mathrm{sd}}^{\mathrm{ij}}$ & Energy-efficiency metric (EEM) of $n_{j}, j \in \mathcal{C}_{s d}^{i}$ \\
\hline $\operatorname{LLM}_{\mathrm{sd}}^{\mathrm{sa}}$ & Low-latency metric (LLM) of $n_{j}, j \in \mathcal{C}_{s d}^{i}$ \\
\hline $\mathcal{R}\left(\mathcal{C}_{s d}^{i}\right)$ & Prioritized $\mathcal{C}_{s d}^{i}$ \\
\hline$F_{X}^{i}\left[\mathcal{C}_{s d}^{i}\right]$ & X-metric fitness of $\mathcal{C}_{s d}^{i}, X \in\{\mathrm{DP}$, EDP, EEM, $\mathrm{LLM}\}$ \\
\hline $\mathcal{S}_{i}$ & Set of nodes in the search space of $n_{i}$ \\
\hline$\psi_{i}^{\star}$ & The best pointing angle of $n_{i}$ \\
\hline $\mathcal{C}_{s d}^{i \star}\left(\psi_{i}\right)$ & The best candidate set at pointi \\
\hline
\end{tabular}

TABLE I: Table of Notations

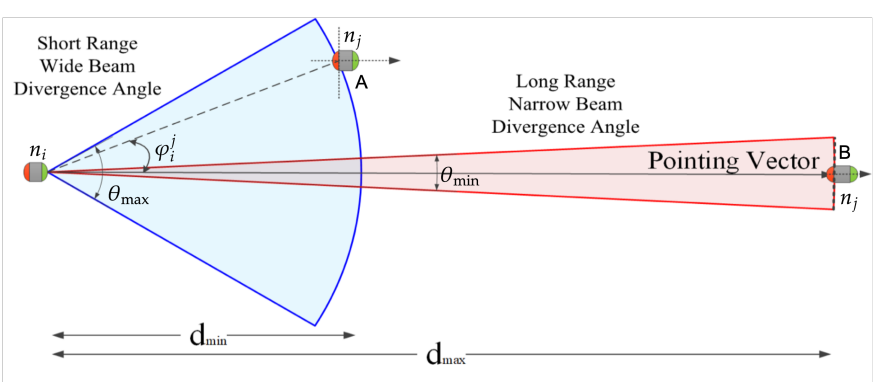

Fig. 2: Illustration of a single-hop link and the tradeoff between divergence angle and communication range.

provide the network with highly connected control links. The sink station is responsible for aggregating data from sensors and disseminating this information to mobile or onshore sinks. Since IoUT data is generally useful only if it is geographically tagged to an accurate sensing location, we assume that each node is aware of its own location $\left(\ell_{i}, i \in[1, \mathrm{M}]\right)$ along with the neighbors within its acoustic communication range. Although underwater location information can be obtained in a fully optical [33]-[35] manner, hybrid opto-acoustic network localization methods are more suitable for the SectOR for two reasons: 1) hybrid methods deliver a better localization accuracy [3], [31] and 2) nodes are already equipped with acoustic transceivers.

\section{B. Channel Model}

According to the Beer's law, absorption and scattering effects of the aquatic medium can be characterized by an extinction coefficient $c(\lambda)=a(\lambda)+b(\lambda)$ where $\lambda, a(\lambda)$, and $b(\lambda)$ denote the carrier wavelength, absorption coefficient, and scattering coefficient, respectively. Based on Beer-Lambert formula, the propagation loss factor between two generic IoUT nodes $n_{i}$ and $n_{j}$ is defined as follows ${ }^{1}$

$$
\mathrm{BL}_{\mathrm{i}}^{\mathrm{j}}=\exp \left\{-c(\lambda) \frac{d_{i}^{j}}{\cos \left(\varphi_{i}^{j}\right)}\right\},
$$

where $d_{i}^{j}$ is the perpendicular distance between the nodes and $\varphi_{i}^{j}$ is the angle between the receiver plane and the transmitterreceiver trajectory, as shown in Fig. 2 where $n_{j}$ is located at point $A$. In case of a perfect alignment, (1) reduces to $\mathrm{BL}_{\mathrm{i}}^{\mathrm{j}}=$ $\exp \left\{-c(\lambda) d_{i}^{j}\right\}$ if $n_{j}$ is located at point $B$. On the other side, the geometrical loss is a result of spreading the light beam to an area larger than the receiver aperture size $A_{j}$ and can be given for a semi-collimated transmitter emitting a Gaussian beam by

$$
\mathrm{GL}_{\mathrm{i}}^{\mathrm{j}}=\left(\frac{A_{j} \cos \left(\varphi_{i}^{j}\right)}{\theta_{1 / e^{i}}^{i} d_{i}^{j}}\right)^{2}
$$

where $\theta_{1 / e}^{i}$ is the is full-width beam divergence angle of $n_{i}$ that is measured at the point where the light intensity drops to $1 / e$ of its peak. In the case of perfect alignment, (2) reduces to the approximation given in [36]. Accordingly, the channel gain between $n_{i}$ and $n_{j}$ is given by the product of (1) and (2) as

$$
\mathrm{G}_{\mathrm{i}}^{\mathrm{j}}=\left(\frac{A_{j} \cos \left(\varphi_{i}^{j}\right)}{\theta_{1 / e}^{i} d_{i}^{j}}\right)^{2} \exp \left\{-c(\lambda) \frac{d_{i}^{j}}{\cos \left(\varphi_{i}^{j}\right)}\right\},
$$

that is merely based on the received ballistic photons which propagate without being disturbed by the scattering effects. That is, (3) neglects all of the scattered photons received by $n_{j}$ by assuming their total loss. By modifying [37. Eq. (4)], scattered rays can be taken into account as follows

$$
\mathrm{G}_{\mathrm{i}}^{\mathrm{j}}=\left(\frac{A_{j} \cos \left(\varphi_{i}^{j}\right)}{\theta_{1 / e^{i} d_{i}^{j}}^{j}}\right)^{2} \exp \left\{-\frac{c(\lambda) d_{i}^{j}}{\cos \left(\varphi_{i}^{j}\right)}\left(\frac{A_{j} \cos \left(\varphi_{i}^{j}\right)}{\theta_{1 / e^{i} d_{i}^{j}}^{j}}\right)^{\alpha}\right\}
$$

where $\alpha$ is a correction coefficient which can be determined based on parameters such as $c(\lambda), A_{j}, \theta_{1 / e}^{i}$, field-of-view (FoV) angle of the receiver, etc.

By analyzing (3) and (4), one can gain valuable insight into the tradeoff between divergence angle and communication range. As illustrated in Fig. 2, a wide divergence angle results in a short communication range so that the source can reach the neighbor nodes within its proximity. On the other hand, a narrow divergence angle helps to reach a distant receiver, which naturally requires an agile and accurate PAT mechanism to sustain a reliable communication link.

\section{Performance Characterization of SINGLE-HOP UNICAST LINKS}

In this section, we characterize the performance of unicast and broadcast links which are used as building blocks of the OR metrics developed in the next section.

\footnotetext{
${ }^{1}$ Throughout this section, we presented channel losses in linear scale Alternatively, they can be expressed in $\mathrm{dB}$ scale by $10 \log _{10}(x)$, where $x$ refers to linear values in (1)-4
} 


\section{A. Unicast Links in TUR}

The source node $n_{s}$ groups messages destined to the destination node $n_{d}$ into packets, each has a length of $L$ bits that consist of a header and a payload. While control messages (e.g., destination address, next forwarder, ACK signaling, etc.) are included in the header, data is encapsulated in the payload that is extracted and used by IoUT applications. TUR paths are formed by consecutive unicast links, i.e., data is forwarded to a unique node at each hop. Thus, we first characterize the performance of a unicast link in terms of distance, reliability, and achievable rates.

Let us consider an arbitrary multihop path between $n_{s}$ and $n_{d}, s \leadsto d=\{s, \ldots, i, j, \ldots, d\}$. Assuming that the number of photons follows a Poisson Process, photon arrival rate from $n_{i}$ to $n_{j}$ is given by [38]

$$
f_{i j}=\frac{P_{r x}^{j} \eta_{c}^{j} \lambda}{R_{i}^{j} \mathrm{~T} \hbar c},
$$

where $\mathrm{P}_{\mathrm{rx}}^{\mathrm{j}}=\mathrm{P}_{\mathrm{tx}}^{\mathrm{i}} \eta_{\mathrm{tx}}^{\mathrm{i}} \eta_{\mathrm{rx}}^{\mathrm{j}} \mathrm{G}_{\mathrm{i}}^{\mathrm{j}}$ is the received power by $n_{j}$, $\mathrm{P}_{\mathrm{tx}}^{\mathrm{i}}$ is the transmission power of $n_{i}$, is the detector counting efficiency of $n_{j}, \mathrm{R}_{\mathrm{i}}^{\mathrm{j}}$ is the data rate, $\mathrm{T}$ is pulse duration, $\hbar$ is Planck's constant, and $c$ is the underwater speed of light. As per the central limit theorem, the Poisson distribution can be approximated by a Gaussian distribution if the number of received photons is large enough. For intensity-modulation/direct-detection (IM/DD) with on-off keying (OOK) modulation, bit error rate (BER) of the link between $n_{i}$ and $n_{j}$ is given by [6]

$$
\overrightarrow{\mathrm{BER}}_{i}^{j}=\frac{1}{2} \operatorname{erfc}\left(\sqrt{\frac{\mathrm{T}}{2}}\left(\sqrt{\mathrm{f}_{\mathrm{ij}}^{1}}-\sqrt{\mathrm{f}_{\mathrm{ij}}^{0}}\right)\right)
$$

where $\operatorname{erfc}(\cdot)$ is the complementary error function, $\mathrm{f}_{\mathrm{ij}}^{1}=\mathrm{f}_{\mathrm{ij}}+$ $\mathrm{f}_{\mathrm{dc}}+\mathrm{f}_{\mathrm{bg}}$ and $\mathrm{f}_{\mathrm{ij}}^{0}=\mathrm{f}_{\mathrm{dc}}+\mathrm{f}_{\mathrm{bg}}$ are the numbers of photon arrivals when binary 1 and binary 0 are transmitted, respectively, $f_{\mathrm{dc}}$ is the additive noise due to dark counts, and $\mathrm{f}_{\mathrm{bg}}$ is the background illumination noise. Accordingly, the packet error rate (PER) and packet delivery ratio (PDR) can be given by

$$
\begin{aligned}
\overrightarrow{\mathrm{PER}}_{i}^{j} & =1-\left(1-\overrightarrow{\mathrm{BER}}_{i}^{j}\right)^{\mathrm{L}} \text { and } \\
\overrightarrow{\mathrm{PDR}}_{i}^{j} & =1-\overrightarrow{\mathrm{PER}}_{i}^{j}=\left(1-\overrightarrow{\mathrm{BER}}_{i}^{j}\right)^{\mathrm{L}},
\end{aligned}
$$

respectively. For a given PER, data rate between $n_{i}$ and $n_{j}$ is then derived by using (5)-(7) as

$$
\mathrm{R}_{\mathrm{i}}^{\mathrm{j}}=\frac{\eta_{\mathrm{j}}^{\mathrm{c}} \lambda}{2 \hbar \mathrm{c}}\left[\frac{\sqrt{\mathrm{P}_{\mathrm{rx}}^{\mathrm{j}}+\mathrm{P}_{\mathrm{dc}}+\mathrm{P}_{\mathrm{bg}}}-\sqrt{\mathrm{P}_{\mathrm{dc}}+\mathrm{P}_{\mathrm{bg}}}}{\operatorname{erfc}^{-1}(2 \mathrm{a})}\right]^{2}
$$

where $\mathrm{P}_{\mathrm{dc}}$ is the dark count noise power, $\mathrm{P}_{\mathrm{bg}}$ is the background noise power, and $a=1-\left(1-\overrightarrow{\mathrm{PER}}_{i}^{j}\right)^{\frac{\mathrm{T}}{\mathrm{L}}}$. For a given data rate $\mathrm{R}_{\mathrm{i}}^{\mathrm{j}}$ and PER $\mathrm{PER}_{\mathrm{i}}^{\mathrm{j}}$, the communication range from $n_{i}$ and toward the direction of $n_{j}$ is obtained as a function of the divergence angle $\theta_{1 / e}^{i}$ by using (3)-9) as follows

$$
D_{\mathrm{i}}^{\mathrm{j}}\left(\theta_{1 / \mathrm{e}}^{\mathrm{i}}\right)=\frac{\left(\frac{2}{(\alpha-1) \mathrm{b}_{3}} \mathrm{~W}_{0}\left[\frac{(\alpha-1)\left(\mathrm{b}_{1} \mathrm{~b}_{2}\right)^{\frac{\alpha-1}{2}} \mathrm{~b}_{3}}{2}\right]\right)^{\frac{1}{1-\alpha}}}{\cos \left(\varphi_{\mathrm{i}}^{\mathrm{j}}\right)},
$$

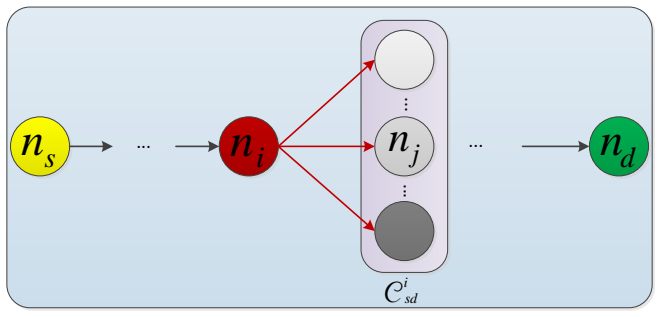

Fig. 3: Illustration of opportunistic routing and broadcast links.

where $W_{0}(\cdot)$ is the principal branch of product logarithm,

$$
\mathrm{b}_{1}=\frac{\left[\sqrt{\frac{2 \mathrm{R}_{\mathrm{i}}^{\mathrm{j}} \hbar \mathrm{c}}{\eta_{\mathrm{j}}^{\mathrm{c} \lambda}}} \operatorname{erfc}^{-1}(2 \mathrm{a})+\sqrt{\mathrm{P}_{\mathrm{dc}}+\mathrm{P}_{\mathrm{bg}}}\right]^{2}-\mathrm{P}_{\mathrm{dc}}-\mathrm{P}_{\mathrm{bg}}}{\mathrm{P}_{\mathrm{tx}}^{\mathrm{i}} \eta_{\mathrm{tx}}^{\mathrm{i}} \eta_{\mathrm{rx}}^{\mathrm{j}}},
$$

$\left.\mathrm{b}_{2}=\left(\frac{\theta_{1 / \mathrm{e}}^{\mathrm{i}}}{\mathrm{A}_{\mathrm{j}} \cos \left(\varphi_{\mathrm{i}}^{\mathrm{j}}\right.}\right)\right)^{2}$, and $\mathrm{b}_{3}=-\frac{\mathrm{c}(\lambda)}{\cos \left(\varphi_{\mathrm{i}}^{\mathrm{j}}\right)}\left(\frac{\mathrm{A}_{\mathrm{j}} \cos \left(\varphi_{\mathrm{i}}^{\mathrm{j}}\right)}{\theta_{1 / \mathrm{e}}^{\mathrm{i}}}\right)^{\alpha}$.

At this point, it is important to relate the previously discussed range $\leftrightarrow$ beamwidth tradeoff with the rate $\leftrightarrow$ reliability one. While the former is specific to OWC since link distance reduces as the divergence angle increases, the latter is common for any communication systems as data rate and PDR are inversely proportional to each other, which follows from (9). Following from (10), these two tradeoffs are also coupled as the range is a function of rate, PDR, and divergence angle.

Assuming that the packet is dropped after $\mathrm{K}$ retransmission attempts, probability of having a successful transmission to $n_{j}$ in $k$ delivery attempts is given by

$$
\mathcal{P}_{i}^{j}(k)=\left(\overrightarrow{\mathrm{PER}}_{i}^{j}\right)^{k-1} \overrightarrow{\mathrm{PDR}}_{i}^{j}, k \in[1, \mathrm{~N}]
$$

Hence, expected number of transmissions (ExNT) can be obtained as

$$
\mathrm{N}_{\mathrm{i}}^{\mathrm{j}}=\sum_{k=1}^{\mathrm{K}} k \mathcal{P}_{i}^{j}(k)+\mathrm{K}\left(\overrightarrow{\mathrm{PER}}_{\mathrm{i}}^{\mathrm{j}}\right)^{\mathrm{K}}
$$

where $\left(\overrightarrow{\mathrm{PER}}_{i}^{j}\right)^{\mathrm{K}}$ is the probability of dropping the package after $\mathrm{K}$ unsuccessful transmissions. If the ExNT is normalized to the probability of having a successful transmission within $\mathrm{K}$ retransmissions, we obtain unicast ExNT as

$$
\overline{\mathrm{N}}_{i}^{j}=\frac{\overrightarrow{\mathrm{N}}_{i}^{j}}{\sum_{k=1}^{\mathrm{K}} \mathcal{P}_{i}^{j}(k)}=\frac{1}{\overrightarrow{\mathrm{PDR}}_{i}^{j}},
$$

which is independent of $\mathrm{N}$.

\section{B. Broadcast Links in $O R$}

Unlike the TUR protocols, OR selects a candidate set (CS) that can overhear the broadcast packets and forward them to the next-hop in a prioritized and coordinated manner. Assuming that $n_{i}$ is one of the forwarder nodes from $n_{s}$ to $n_{d}$ [c.f. Fig. 3], $\mathcal{C}_{s d}^{i}$ is denoted as the candidate index set of the forwarder node $n]^{2}$. For simplicity, we assume that $\mathcal{C}_{s d}^{i}$ is

\footnotetext{
${ }^{2} \mathcal{C}_{s d}^{i}$ may also include the destination node if it is within the communication range.
} 
ordered in the descending order of forwarding priority. That is, the $k^{t h}$ member of $\mathcal{C}_{s d}^{i}$ attempts to forward packet only if the $j^{t h}$ member fails, $k>j$. In this case, packet delivery fails if none of the CS members successfully receives the packet, i.e.,

$$
\mathrm{PER}_{\mathrm{sd}}^{\mathrm{i}}=\prod_{j \in \mathcal{C}_{\mathrm{sd}}^{\mathrm{i}}} \overrightarrow{\mathrm{PER}}_{\mathrm{i}}^{\mathrm{j}} .
$$

Accordingly, successful packet forwarding ratio is obtained as

$$
\mathrm{SFR}_{\mathrm{sd}}^{\mathrm{ij}}=\overrightarrow{\mathrm{PDR}}_{\mathrm{i}}^{\mathrm{j}} \prod_{\mathrm{k}=1}^{\mathrm{j}-1} \overrightarrow{\mathrm{PER}}_{\mathrm{i}}^{\mathrm{k}}
$$

which is the probability that $n_{j}$ successfully receives the packet from $n_{i}$ given that higher priority candidates $\mathcal{C}_{s d}^{i k}$, $k<j$, fail. Hence, ExNT in the OR scheme is given by

$$
\mathrm{N}_{\mathrm{sd}}^{\mathrm{i}}=\sum_{\mathrm{k}=1}^{\mathrm{K}} \mathrm{k}\left(\mathrm{PER}_{\mathrm{sd}}^{\mathrm{i}}\right)^{\mathrm{k}-1} \mathrm{PDR}_{\mathrm{sd}}^{\mathrm{i}}+\mathrm{K}\left(\mathrm{PER}_{\mathrm{sd}}^{\mathrm{i}}\right)^{\mathrm{K}}
$$

where the first term is the ExNT for successfully delivering a package to $n_{j}$ and the second term accounts for the package drop event. As in (13), the ExNT normalized to the success probability is given by

$$
\overline{\mathrm{N}}_{s d}^{i}=\frac{1}{\mathrm{PDR}_{\mathrm{sd}}^{\mathrm{i}}}=\frac{1}{1-\prod_{j \in \mathcal{C}_{s d}^{i}} \overline{\mathrm{PER}}_{i}^{j}} .
$$

which is referred to as broadcast ExNT in the rest of the paper.

\section{Opportunistic Routing Metrics}

OR metrics play a crucial role in the performance of the routing protocol since it has a direct impact on the candidate selection and prioritization outcomes. Based on the available network state information at each node, the OR metrics can be classified into local and global metrics, which require information from neighboring nodes and the entire network topology, respectively. We denote $\Varangle\left(d, \theta_{1 / e}^{i}, \psi_{i}\right)$ as the sectorshaped coverage region of $n_{i}$ with divergence angle $\theta_{1 / e}^{i}$ centered at the pointing angle $\psi_{i}$. Notice that one can alter the sector-shaped optical coverage area of $n_{i}$ by changing $\psi_{i}$ and $\theta_{1 / e}^{i}$. That is, the elements of $\mathcal{C}_{s d}^{i}\left(\psi_{i}, \theta_{1 / e}^{i}\right)$ vary with different pairs of $\psi_{i}$ and $\theta_{1 / e}^{i}$. For the sake of clarity, we omit these parameters from $\mathcal{C}_{s d}^{i}\left(\psi_{i}, \theta_{1 / e}^{i}\right)$, and focus our attention on a single CS, $\mathcal{C}_{s d}^{i}$, throughout this section. Next, we present local and global OR metrics that account for different routing objectives.

\section{A. Local Opportunistic Routing (LOR) Metrics}

Local OR metrics are generally preferred to avoid the cost of updating and storing the entire topology state. We assume that each node has the location information of itself, onehop neighborhood (i.e., acoustic coverage), and the destination (i.e., the sink). In what follows, we introduce local OR metrics for distance progress, energy consumption, and delay.
1) Distance Progress: One of the most common local OR metrics is the distance progress (DP), which selects and orders the candidate according to their closeness toward the destination [39]. The DP metric for $n_{j} \in \mathcal{C}_{s d}^{i}$ is given as

$$
\mathrm{DP}_{\mathrm{sd}}^{\mathrm{ij}}=\left(\left\|\ell_{\mathrm{s}}-\ell_{\mathrm{d}}\right\|-\left\|\ell_{\mathrm{j}}-\boldsymbol{\ell}_{\mathrm{d}}\right\|\right), \forall \mathrm{j} \in \mathcal{C}_{\mathrm{sd}}^{\mathrm{i}}
$$

Accordingly, the prioritized $\mathcal{C}_{s d}^{i}$ for DP metric is given by

$$
\mathcal{R}\left(\mathcal{C}_{s d}^{i}\right)=\left\{j \mid \mathrm{DP}_{\mathrm{sd}}^{\mathrm{ij}}>\mathrm{DP}_{\mathrm{sd}}^{\mathrm{il}}, \mathrm{j}<\mathrm{l}, \forall(\mathrm{j}, \mathrm{l}) \in \mathcal{C}_{\mathrm{sd}}^{\mathrm{i}}\right\} .
$$

Lastly, the DP fitness of $\mathcal{C}_{s d}^{i}$ is given by

$$
\mathrm{F}_{\mathrm{DP}}^{\mathrm{i}}\left[\mathcal{C}_{\mathrm{sd}}^{\mathrm{i}}\right]=\max _{\forall \mathrm{j} \in \mathcal{C}_{\mathrm{sd}}^{\mathrm{i}}}\left\{\mathrm{DP} \mathrm{P}_{\mathrm{sd}}^{\mathrm{ij}}\right\} .
$$

Notice that measuring the OR metric in traveled distance implicitly sets the routing objective to minimize the number of hops. In terrestrial WSNs, the DP metric is limited to the scenario where a very far away candidate is selected merely based on its proximity without accounting for the link quality. Due to the short-range and directed propagation of light in the water, the negative consequences of this limitation can be mitigated by restricting the candidate set to the sectorshaped coverage region. A more advanced version of DP is the expected distance progress (EDP) that considers the average DP [40] by accounting for the link quality. The EDP metric of $n_{i}$ is given by

$$
\mathrm{EDP}_{\mathrm{sd}}^{\mathrm{ij}}=\mathrm{DP}_{\mathrm{sd}}^{\mathrm{ij}} \mathrm{SFR}_{\mathrm{sd}}^{\mathrm{ij}}, \forall \mathrm{j} \in \mathcal{C}_{\mathrm{sd}}^{\mathrm{i}}
$$

which accounts for connectivity, link quality, and distance advancement toward the sink at the same time. Accordingly, the $\mathrm{j}^{\text {th }}$ element of priority set can be iteratively determined as follows

$$
\mathcal{R}_{s d}^{i j}=\left\{\begin{array}{ll}
\underset{k \in \mathcal{C}_{s d}^{i}}{\operatorname{argmax}} & \left(\mathrm{DP}_{\mathrm{sd}}^{\mathrm{ik}} \mathrm{PDR}_{\mathrm{i}}^{\mathrm{k}}\right), j=1 \\
\underset{k \in \mathcal{C}_{s d}^{i}-\cup_{1}^{j-1} \mathcal{R}_{s d}^{i j}}{\operatorname{argmax}} & \left(\mathrm{DP}_{\mathrm{sd}}^{\mathrm{ik}} \mathrm{SFR}_{\mathrm{sd}}^{\mathrm{ik}}\right), j>1
\end{array},\right.
$$

where the first element is determined based on highest individual performance of nodes while the remaining nodes are iteratively determined based on the latest form of the priority set. Lastly, the EDP fitness of $\mathcal{C}_{s d}^{i}$ is given by

$$
\mathrm{F}_{\mathrm{EDP}}^{\mathrm{i}}\left[\mathcal{C}_{\mathrm{sd}}^{\mathrm{i}}\right]=\sum_{\mathrm{j} \in \mathcal{C}_{\mathrm{sd}}^{\mathrm{i}}} \mathrm{DP}_{\mathrm{sd}}^{\mathrm{ij}} \mathrm{SFR}_{\mathrm{sd}}^{\mathrm{ij}} \text {. }
$$

2) Energy Efficiency: Since IoUT nodes operate on limited battery capacity, energy-efficient OR plays a crucial role in UOAN lifetime maximization. Indeed, the consumed energy increases with the number of transmission attempts, each of which costs an energy dissipation as a result of transmission, reception, and coordination. Hence, the energy cost of making $k$ transmission can be formulated as

$$
\mathrm{E}_{\mathrm{i}}(\mathrm{k})=\mathrm{k}\left[\mathrm{T}_{\mathrm{s}}\left(\mathrm{P}_{\mathrm{tx}}^{\mathrm{i}}+\sum_{\mathrm{j} \in \mathcal{C}_{\mathrm{sd}}^{\mathrm{i}}} \mathrm{P}_{\mathrm{l}}^{\mathrm{j}}\right)+\mathrm{P}_{\mathrm{c}} \mathrm{T}_{\mathrm{c}}^{\mathrm{i}}\right],
$$

where $T_{s}=\frac{L}{R_{i}}$ is the transmission duration, $P_{l}^{j}$ is the listening power consumed by decoding and signal processing circuitry, and $P_{c}$ is the coordination power consumed by candidates 
during the coordination duration $T_{d}^{\sqrt[3]{3}}$ Based on (24), the energy efficiency metric (EEM) for $n_{j}$ is given by

$$
\operatorname{EEM}_{\mathrm{sd}}^{\mathrm{ij}}=\mathrm{E}_{\mathrm{i}}\left(\mathrm{N}_{\mathrm{i}}^{\mathrm{j}}\right)
$$

where $\mathrm{N}_{\mathrm{i}}^{\mathrm{j}}$ can be obtained by (12). Accordingly, the prioritized $\mathcal{C}_{s d}^{i}$ for EEM metric is given by

$$
\mathcal{R}\left(\mathcal{C}_{s d}^{i}\right)=\left\{j \mid \operatorname{EEM}_{\mathrm{sd}}^{\mathrm{jk}}<\operatorname{EEM}_{\mathrm{sd}}^{\mathrm{il}}, \mathrm{j}<\mathrm{l}, \forall(\mathrm{j}, \mathrm{l}) \in \mathcal{C}_{\mathrm{sd}}^{\mathrm{i}}\right\} .
$$

Thus, EEM fitness of $\mathcal{C}_{s d}^{i}$ is given by

$$
\mathrm{F}_{\text {EEM }}^{\mathrm{i}}\left[\mathcal{C}_{\mathrm{sd}}^{\mathrm{i}}\right]=\mathrm{E}_{\mathrm{i}}\left(\mathrm{N}_{\mathrm{sd}}^{\mathrm{i}}\right) \text {. }
$$

where $\mathrm{N}_{\mathrm{sd}}^{\mathrm{i}}$ is the broadcast ExNT given in (17).

3) Low Latency: Latency is a critical metric, especially for delay intolerant underwater applications. Similar to EEM, low latency metric (LLM) increases with the number of transmission attempts, each of which costs a delay due to the transmission and coordination among the candidate nodes. Hence, the delay caused by $n$ transmission attempts can be formulated as

$$
\mathrm{D}_{\mathrm{i}}(\mathrm{k})=\mathrm{k}\left[\mathrm{T}_{\mathrm{s}}+\mathrm{T}_{\mathrm{c}}^{\mathrm{i}}\right],
$$

which can be used to calculate the LLM of $n_{j}$ as follows

$$
\operatorname{LLM}_{\mathrm{sd}}^{\mathrm{ij}}=\mathrm{D}_{\mathrm{i}}\left(\mathrm{N}_{\mathrm{i}}^{\mathrm{j}}\right) \text {. }
$$

Similarly, the prioritized $\mathcal{C}_{s d}^{i}$ for LLM metric is given by

$$
\mathcal{R}\left(\mathcal{C}_{s d}^{i}\right)=\left\{j \mid \operatorname{LLM}_{\mathrm{sd}}^{\mathrm{jk}}<\operatorname{LLM}_{\mathrm{sd}}^{\mathrm{il}}, \mathrm{j}<\mathrm{l}, \forall(\mathrm{j}, \mathrm{l}) \in \mathcal{C}_{\mathrm{sd}}^{\mathrm{i}}\right\} .
$$

Lastly, the LLM fitness of $\mathcal{C}_{s d}^{i}$ is given by

$$
\mathrm{F}_{\text {LLM }}^{\mathrm{i}}\left[\mathcal{C}_{\mathrm{sd}}^{\mathrm{i}}\right]=\mathrm{D}_{\mathrm{i}}\left(\mathrm{N}_{\mathrm{sd}}^{\mathrm{i}}\right) \text {. }
$$

where $\mathrm{N}_{\mathrm{sd}}^{\mathrm{i}}$ is the broadcast ExNT given in (17).

\section{B. Global Opportunistic Routing (GOR) Metrics}

The main objective of the global OR metric is to reduce the ExNT such that end-to-end (E2E) ExNT, energy consumption, and delay is minimized. Since global OR metrics capture the ExNT while taking all possible multipath, they are generally expressed in recursive formulas. Naturally, these require a massive control signaling overhead and computational power.

For a forwarder node $n_{i}$, an E2E metric can be obtained by summation of two components: 1) The metric from $n_{i}$ to its $\mathrm{CSC}_{s d}^{i}$ and 2) The metric from its candidates to the destination node, $n_{d}$. In this case, we can rewrite the global version of 24) in a recursive form as follows

$$
\mathrm{E}_{\mathrm{i}}^{\mathrm{j}}(\mathrm{k})=\mathrm{E}_{\mathrm{i}}(\mathrm{k})+\mathrm{E}_{\mathrm{j}}\left(\mathrm{N}_{\mathrm{sd}}^{\mathrm{j}}\right),
$$

$\mathrm{N}_{\mathrm{sd}}^{\mathrm{j}}$ is given in 16 . Correspondingly, the probability that transmission is failed in previous $k-1$ attempts and $n_{j}$ is successfully received the packet at the $k^{t h}$ attemp ${ }^{4}$ is given by

$$
\mathcal{P}_{s d}^{i j}(k)=\left\{\begin{array}{ll}
\left(\mathrm{PER}_{\mathrm{sd}}^{\mathrm{i}}\right)^{k-1} \overrightarrow{\mathrm{PDR}}_{i}^{d} & , n_{j}=n_{d} \\
\left(\mathrm{PER}_{\mathrm{sd}}^{\mathrm{i}}\right)^{k-1} \mathrm{SFR}_{\mathrm{sd}}^{\mathrm{ij}} & , \text { otherwise }
\end{array} .\right.
$$

\footnotetext{
${ }^{3}$ We revisit the components of $T_{c}$ during the candidate coordination discussion in Section $\mathrm{V}-\mathrm{C}$

${ }^{4}$ To make this happen, nodes with a priority higher than $n_{j}$ must also fail in the $n^{\text {th }}$ attempt.
}

Finally, the EEM fitness of $n_{i}$ is derived as

$$
\mathrm{F}_{\mathrm{EEM}}^{\mathrm{i}}\left[\mathcal{C}_{\mathrm{sd}}^{\mathrm{i}}\right]=\sum_{\mathrm{j} \in \mathcal{C}_{\text {sd }}^{\mathrm{i}}} \sum_{\mathrm{k}=1}^{\mathrm{K}} \mathrm{E}_{\mathrm{i}}^{\mathrm{j}}(\mathrm{k}) \mathcal{P}_{\mathrm{sd}}^{\mathrm{ij}}(\mathrm{k})+\mathrm{E}_{\mathrm{i}}(\mathrm{K})\left(\operatorname{PER}_{\mathrm{sd}}^{\mathrm{i}}\right)^{\mathrm{K}}
$$

which is the expected total energy cost of reaching to the destination node through the forwarders in $\mathcal{C}_{s d}^{i}$ Following from (34), the candidates can be prioritized by their energy consumption towards the destination node as follows

$$
\mathcal{R}\left(\mathcal{C}_{s d}^{i}\right)=\left\{j \mid \operatorname{EEM}_{\mathrm{sd}}^{\mathrm{j}}<\operatorname{EEM}_{\mathrm{sd}}^{\mathrm{l}}, \mathrm{j}<\mathrm{l}, \forall(\mathrm{j}, \mathrm{l}) \in \mathcal{C}_{\mathrm{sd}}^{\mathrm{i}}\right\} .
$$

Similar to (32), we can rewrite global version of 28 in a recursive form as

$$
\mathrm{D}_{\mathrm{i}}^{\mathrm{j}}(\mathrm{n})=\mathrm{D}_{\mathrm{i}}(\mathrm{n})+\mathrm{D}_{\mathrm{j}}\left(\mathrm{N}_{\mathrm{sd}}^{\mathrm{j}}\right),
$$

which then can be used to calculate the LLM fitness of $n_{i}$ as

$$
\mathrm{F}_{\mathrm{LLM}}^{\mathrm{i}}\left[\mathcal{C}_{\mathrm{sd}}^{\mathrm{i}}\right]=\sum_{\mathrm{j} \in \mathcal{C}_{\mathrm{sd}}^{\mathrm{i}}} \sum_{\mathrm{k}=1}^{\mathrm{K}} \mathrm{D}_{\mathrm{i}}^{\mathrm{j}}(\mathrm{k}) \mathcal{P}_{\mathrm{sd}}^{\mathrm{ij}}(\mathrm{k})+\mathrm{D}_{\mathrm{i}}(\mathrm{K})\left(\operatorname{PER}_{\mathrm{sd}}^{\mathrm{i}}\right)^{\mathrm{K}}
$$

which is the expected total delay to reach the destination node through the forwarders in $\mathcal{C}_{s d}^{i}$. Following from (37), the candidates can be prioritized by their energy consumption towards the destination node as follows

$$
\mathcal{R}\left(\mathcal{C}_{s d}^{i}\right)=\left\{j \mid \mathrm{LLM}_{\mathrm{sd}}^{\mathrm{j}}<\operatorname{LLM}_{\mathrm{sd}}^{\mathrm{l}}, \mathrm{j}<\mathrm{l}, \forall(\mathrm{j}, \mathrm{l}) \in \mathcal{C}_{\mathrm{sd}}^{\mathrm{i}}\right\} .
$$

These E2E metrics can also be transformed into a global ExNT metric as explained at the end of previous section.

\section{SectOR: Sector-Based Opportunistic Routing}

This section focuses on designing and providing the proposed SectOR protocol that consists of three main components: 1) Candidate Filtering, 2) Candidate Selection, and 3) Candidate Coordination. Then, we provide the algorithmic implementation of the SectOR. Nodes are only interested in discovering neighbors within the optical communication range. Therefore, during these steps, we also limit the acoustic range to the maximum optical range $d_{\max }$, which is obtained at minimum divergence angle $\theta_{\min }$. In this way, nodes can adjust the acoustic system's transmission power to eliminate unnecessary power consumption and minimize interference to other nodes. Nonetheless, nodes can still adjust their acoustic transmission power to communicate with distant locations whenever it is necessary.

\section{A. Candidate Filtering}

The sector-shaped optical coverage region changes with two prominent parameters; pointing direction and divergence angle. Thus, candidate filtering determines the search space (SS) where we manipulate these angles to find CS with the best fitness. To provide a better insight into the candidate filtering, let us pictorially explain it with the help of Fig. 4 For a given divergence angle range $\theta_{\min } \leqslant \theta_{1 / e}^{i} \leqslant \theta_{\max }$, the maximum and minimum distance $d_{\max }$ and $d_{\min }$ can be obtained by substituting $\theta_{\min }$ and $\theta_{\max }$ into (10), respectively. Since the CS is to be extracted from the SS, we should filter the SS out 


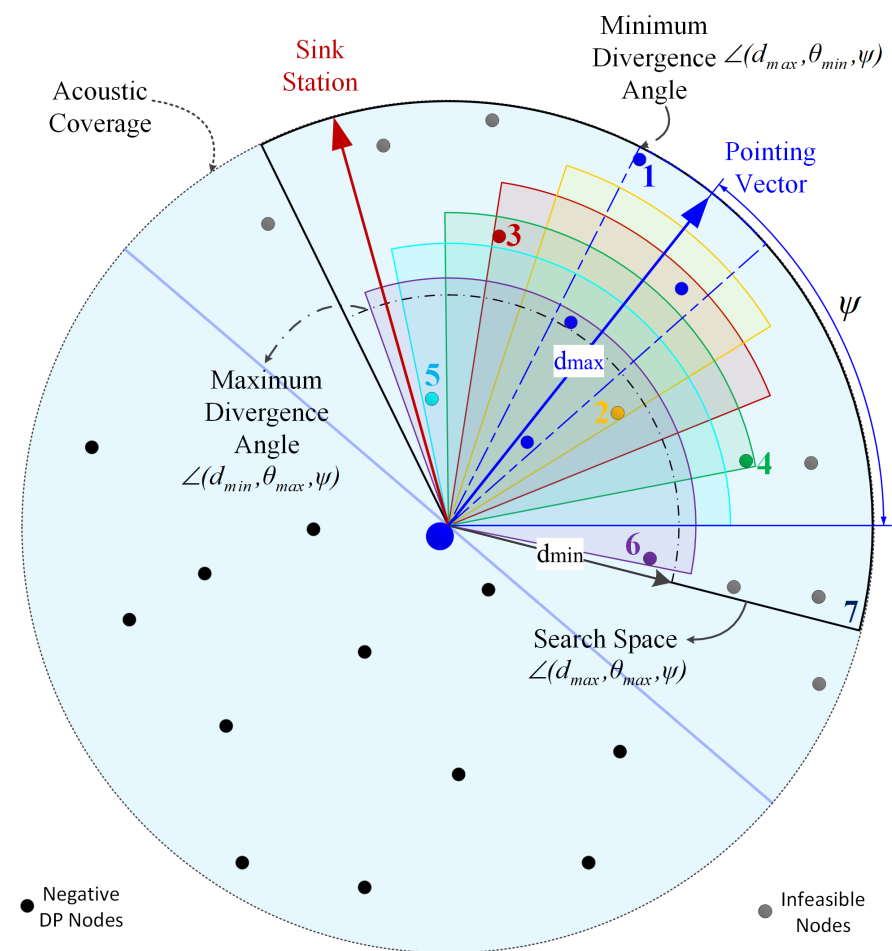

Fig. 4: Illustration of Candidate Filtering and Selection.

of the disk-shaped acoustic communication range. Thus, the $\mathrm{SS}$ of $n_{i}$ is given by

$$
\mathcal{S}_{i}=\left\{\boldsymbol{\ell}_{x} \mid \forall x,\left\|\boldsymbol{\ell}_{x}-\boldsymbol{\ell}_{i}\right\|>d_{\max },\left\|\ell_{i}-\boldsymbol{\ell}_{d}\right\|>\left\|\boldsymbol{\ell}_{x}-\boldsymbol{\ell}_{d}\right\|\right\},
$$

which is the set of locations falls within the coverage region with a positive DP [c.f. Fig. 4]. Notice that positive DP condition is crucial especially for local EEM and LLM metrics, where paths may be routed to wrong directions. Since GOR metrics have the global network view, the SS of GOR metrics are allowed to consider all nodes within the acoustic range, i.e., $\mathcal{S}_{i}=\left\{\boldsymbol{\ell}_{x} \mid \forall x,\left\|\boldsymbol{\ell}_{x}-\boldsymbol{\ell}_{i}\right\|>d_{\max }\right\}$.

In our previous work [1], we adopted the conventional method of fixing pointing vector towards the sink station as in [28], [30]. Here, we allow each node to determine its pointing angle within the SS area. To this end, $n_{i}$ can rotate $\psi_{i}$ in the counter-clockwise direction and record the angle wherever a new node is entered into the SS. The list of these recorded pointing angles of $n_{i}$ is denoted by $\Psi_{i}$. Accordingly, $n_{i}$ evaluates the fitness of each pointing angle as described in the next section and select the best pointing angle $\psi_{i}^{\star}$ as in 39 . These two approaches are illustrated in Fig. 4 where setting the pointing vector towards the sink station is not desirable because it delivers a poor performance.

\section{B. Candidate Selection}

As a result of rate $\leftrightarrow$ reliability and range $\leftrightarrow$ beam-width tradeoffs, the divergence angle has the main impact on feasible candidate sets (colored sectors in Fig. 4) and their performance in terms of the underlying OR metric. For a data and error rate pair, divergence angle determines the range and thus the CS size, which eventually affects DP, EDP, ExNT, energy consumption, delay, etc.

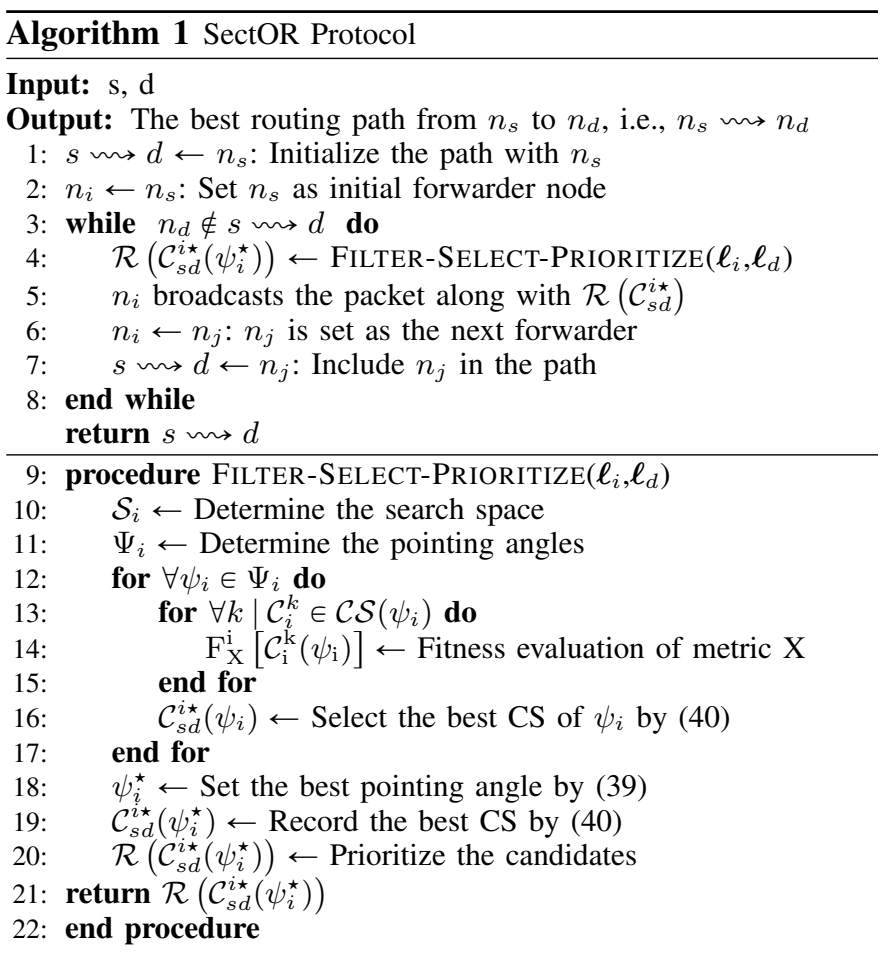

Therefore, SectOR manipulates the range $\leftrightarrow$ beamwidth tradeoff to obtain a CS which delivers the best OR metric. Now let us consider the pointing angle $\psi_{i}^{\star}$ and denote the set of feasible CSs by $\mathcal{C S}\left(\psi_{i}^{\star}\right)=\left\{\mathcal{C}_{i}^{1}\left(\psi_{i}^{\star}\right), \ldots, \mathcal{C}_{i}^{\aleph_{i}}\left(\psi_{i}^{\star}\right)\right\}$ where $\aleph_{i}=\left|\Psi_{i}\right|$. In $\mathcal{C} \mathcal{S}\left(\psi_{i}^{\star}\right)$, the first and the last CSs can be given by $\Varangle\left(d_{\max }, \theta_{\min }, \psi_{i}^{\star}\right)$ and $\Varangle\left(d_{\min }, \theta_{\max }, \psi_{i}^{\star}\right)$ which are shown in black colored dashed and dotted-dashed sectors in Fig. 4 respectively. The rest of CSs may be obtained by quantizing the interval $\theta_{1 / e}^{i} \in\left[\theta_{\min }, \theta_{\max }\right]$ which may be computationally complex for higher resolution. However, this complexity can be augmented by selecting only necessary quantization points based on the node locations within the SS. As shown by colored sectors in Fig. 4. we widen $\theta_{1 / e}^{i}$ starting from $\theta_{\text {min }}$ up to $\theta_{\max }$ and create a new CS whenever a new node is covered by the sector shaped coverage region. Fig. 4 illustrates this simple yet accurate approach by 7 colored sectors among which sectors, i.e., CSs, identified with this approach are obtained by nodes numbered between 2 and 6 , as 1 and 6 are the sectors at minimum and maximum divergence angle, respectively. In this way, all CS combinations are considered without complications caused by an exhaustive quantization approach. Accordingly, the best CS of $n_{i}$ is determined by (40). We must note that a node cannot belong to the CS at two different hops. That is, CSs do not overlap and hence interference between the consecutive hops is not possible.

\section{Candidate Coordination}

For the candidate coordination, we consider an acknowledgment (ACK) based method where candidates return ACK messages in the priority order embedded in the received packet's header. Slotted Acknowledgment (SA) is one of the first coordination methods [41] where each candidate sends its ACK with a delay of short interframe space (SIFS) 


$$
\begin{gathered}
\psi_{i}^{\star}=\left\{\begin{array}{l}
\max _{\psi \in \Psi_{i}}\left(\mathrm{~F}_{\mathrm{X}}^{\mathrm{i}}\left[\mathcal{C}_{\mathrm{sd}}^{\mathrm{i} \star}\left(\psi_{\mathrm{i}}\right)\right]\right), \text { for DP, EDP } \\
\min _{\psi \in \Psi_{i}}\left(\mathrm{~F}_{\mathrm{X}}^{\mathrm{i}}\left[\mathcal{C}_{\mathrm{sd}}^{\mathrm{i} \star}\left(\psi_{\mathrm{i}}\right)\right]\right), \text { otherwise }
\end{array}\right. \\
\mathcal{C}_{s d}^{i \star}\left(\psi_{i}\right)=\left\{\begin{array}{l}
\left\{\mathcal{C}_{i}^{k}\left(\psi_{i}\right) \mid k=\underset{1 \leqslant \ell \leqslant \aleph_{i}}{\operatorname{argmax}}\left\{\mathrm{F}_{\mathrm{X}}^{\mathrm{i}}\left[\mathcal{C}_{\mathrm{i}}^{\ell}\left(\psi_{\mathrm{i}}\right)\right]\right\}\right\}, \mathrm{X} \in\{\mathrm{DP}, \mathrm{EDP}\} \\
\left\{\mathcal{C}_{i}^{k}\left(\psi_{i}\right) \mid k=\underset{1 \leqslant \ell \leqslant \aleph_{i}}{\operatorname{argmin}}\left\{\mathrm{F}_{\mathrm{X}}^{\mathrm{i}}\left[\mathcal{C}_{\mathrm{i}}^{\ell}\left(\psi_{\mathrm{i}}\right)\right]\right\}\right\}, \text { otherwise }
\end{array}\right.
\end{gathered}
$$

duration, $\tau_{S I F S}$, which yields a coordination delay of $T_{c}^{i}=$ $C_{s d}^{i}\left(\tau_{S I F S}+\tau_{A C K}\right)$, where $C_{s d}^{i}=\left|\mathcal{C}_{s d}^{i}\right|$ and $\tau_{A C K}$ is the duration of ACK signal. Since SA is vulnerable to the collision of data and ACK packages, a compressed slotted acknowledgment (CSA) method is developed by means of channel sensing techniques [42] that has a coordination delay of $T_{c}^{i}=\tau_{S I F S}+C_{s d}^{i} \tau_{A C K}$.

We must note that SectOR is not vulnerable to the collision between data and ACK packages since acoustic links can be used to exchange ACK messages in case of collisions or optical link failures. Whenever there is a need for using acoustic modem to transmit control messages, the network interface directly communicate with the acoustic modem to send commands out. Indeed, one can expect acoustic control links to reach a high success probability since the ACK messaging occurs within the UOWC range, which is far less than the acoustic range. Moreover, it is worth mentioning that on-demand involvement of low-rate acoustic links is beneficial to avoid high power consumption and delay.

However, SectOR still needs to account for data transmission of multiple candidates. Hence, we adopt fast slotted acknowledgment (FSA) [43], where each candidate waits before deciding whether it should broadcast ACK. This waiting duration is given by $\tau_{S I F S}+(k-1) \tau_{\text {sens }}$ where $\tau_{\text {sens }}$ is the acoustic channel sensing duration, and $k$ is the priority order in the candidate set. Accordingly, FSA eliminates the possibility of data packet collision at the cost of $T_{c}^{i}=\tau_{S I F S}+\tau_{A C K}+$ $C_{s d}^{i} \tau_{\text {sens }}$ coordination delay. Noting that $\tau_{\text {sens }}$ is negligible in comparison with other terms, FSA requires only a single pair of $\tau_{S I F S}$ and $\tau_{A C K}$. We refer interested readers to [43] for a more in-depth discussion about coordination delay comparison among SA, CSA, and FSA methods.

\section{Algorithmic Implementation of SectOR}

The algorithmic implementation of primarily depends on the FILTER-SELECT-PRIORITIZE $\left(\ell_{i}\right)$ procedure, where $n_{i}, \forall i$, execute the candidate filtering, selection, and prioritization procedure. We should note that SectOR is also suitable for underwater mobile networks since this procedure is independently repeated by each node if there is a change in their SS, i.e., neighborhood. In this procedure, lines 10 and 11 calculate the SS, $\mathcal{S}_{i}$, and set of pointing angles, $\Psi_{i}$, based on $\ell_{i}$, respectively. Then, the nested for loops between lines 12 and 17 compute OR metrics, evaluates fitness functions, and select the best CS, $\forall \psi_{i} \in \Psi_{i}$. Line 18 sets the best pointing angle using (39), then corresponding CSs are calculated using
TABLE II: Table of Parameters

\begin{tabular}{|l|l|l|l|l|l|}
\hline Par. & Value & Par. & Value & Par. & Value \\
\hline$P t x$ & $0.1 \mathrm{~W}$ & $\hbar$ & $6.62 \mathrm{E}-34$ & $T$ & $1 \mathrm{~ns}$ \\
\hline$\eta_{x}$ & 0.9 & $c$ & $2.55 \mathrm{E} 8 \mathrm{~m} / \mathrm{s}$ & $R$ & $1 \mathrm{Gbps}$ \\
\hline$\eta_{r}$ & 0.9 & $\lambda$ & $532 \mathrm{E}-9$ & $L$ & $124 \mathrm{~B}$ \\
\hline$\eta_{c}$ & 0.16 & $e(\lambda)$ & 0.1514 & $\overline{\mathrm{PER}}$ & 0.1 \\
\hline$A$ & $5 \mathrm{~cm}^{2}$ & $f_{b g}$ & $1 \mathrm{E} 6$ & $f_{d c}$ & $1 \mathrm{E} 6$ \\
\hline$\delta$ & 0.01 & $\theta_{\min }$ & $0.336 \mathrm{rad}$ & $\theta_{\max }$ & $2 / 3 \mathrm{rad}$ \\
\hline
\end{tabular}

(40) in line 19. Lines 20 and 21 prioritize the elements of $\mathcal{C}_{s d}^{i \star}$ and return the prioritized candidate set, respectively.

Notice that the SectOR is a fully distributed routing protocol as each node maintains its own prioritized CS. Once a node receives a packet, it independently operates to forward received packets based on up-to-date prioritized CS. At this point, the underlying OR metric type plays a vital role in the optimality of the final route to the destination. If nodes use global OR metrics to determine their prioritized CSs, the resulting route is optimal at the cost of more computational power and communication overhead. Although using global OR metrics may not be practical under real-life conditions, we use them as a benchmark to evaluate local OR metrics' performance.

In light of above discussions, algorithmic implementation of SectOR protocol is summarized between lines 1 and 8 of Algorithm 11. which first initializes the path $n_{s} \leadsto n_{d}$ and current forwarder node to $n_{s}$. Until $n_{d}$ is reached, while loop between lines 3 and 8 repeats the following procedure: The current forwarder node $n_{i}$ broadcasts the packet with the header along with the destination node and prioritized CS. After $T_{c}^{i}$ coordination duration, $n_{j}$ is selected to forward the data packet received from $n_{i}$. Thereafter, $n_{j}$ independently operates from $n_{i}$ to deliver the received packet to the destination node. It is worth noting that all OR metrics presented in this paper is additive due to the additivity of distance and ExNT. Therefore, the end-to-end fitness of routing path $s \leadsto d$ to an OR metrix $X$ can be calculated by

$$
\mathrm{F}_{\mathrm{s} m \mathrm{~d}}=\sum_{\mathrm{i} \in \mathrm{s} m \mathrm{~d}} \mathrm{~F}_{\mathrm{X}}^{\mathrm{i}}\left[\mathcal{C}_{\mathrm{sd}}^{\mathrm{i} \star}\left(\psi_{\mathrm{i}}^{\star}\right)\right]
$$

where $\mathrm{X} \in\{\mathrm{DP}, \mathrm{EDP}, \mathrm{EEM}, \mathrm{LLM}\}$.

\section{NUMERICAL RESULTS}

We consider a square network area with varying side lengths (SL). Throughout simulations, the source and the sink nodes are located at reference points of $(0,0)$ and $(\mathrm{SL}, \mathrm{SL})$, respectively. The remaining 50 nodes are uniformly distributed over the SL $\times \mathrm{SL} m^{2}$ network area. Obtained results are averaged over 10,000 random realizations. Unless it is stated 


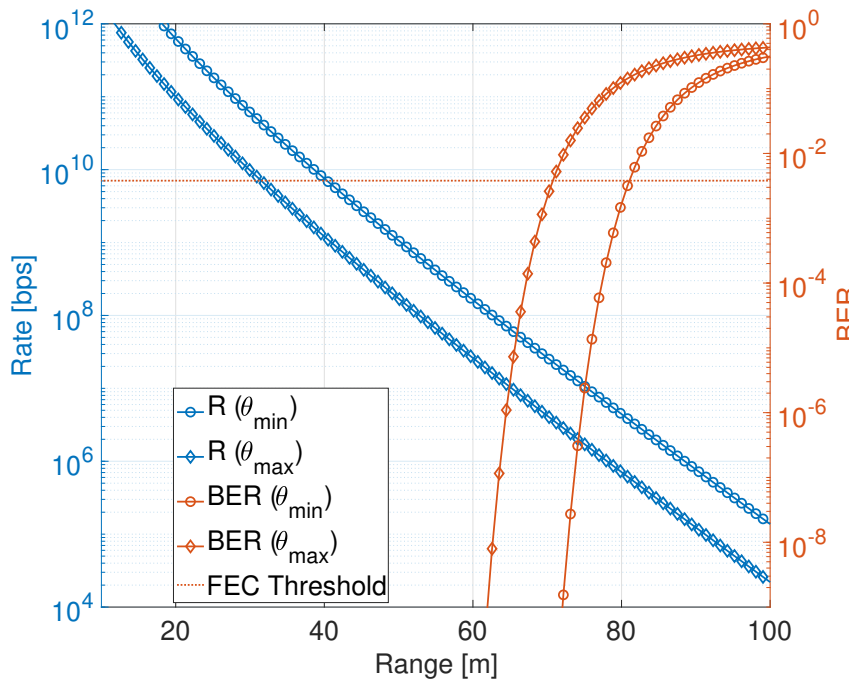

(a) Range vs. Rate and BER. (BER=FEC, R=1 Mbps)

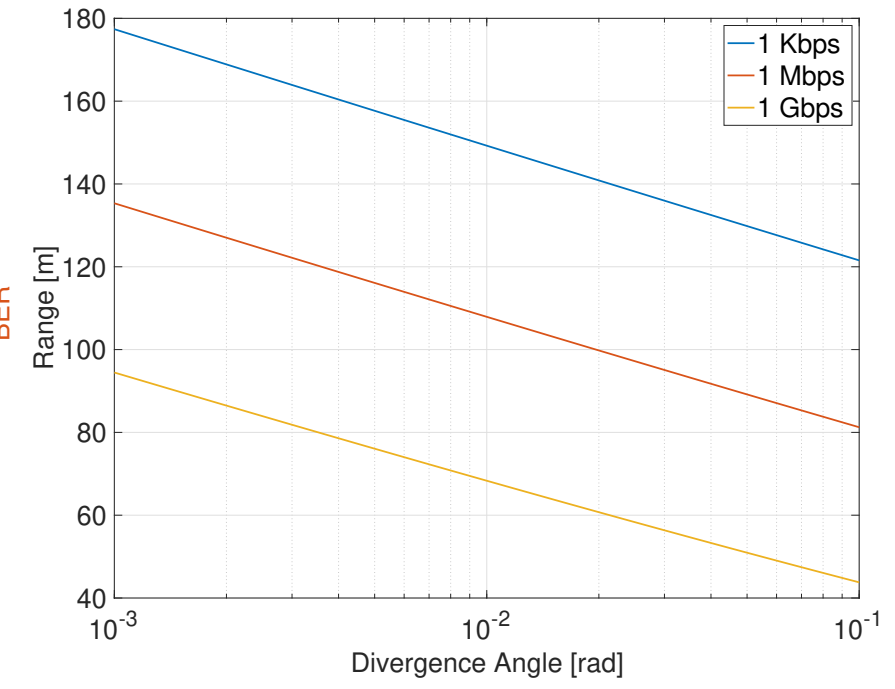

(b) Range vs. divergence angle. (BER=FEC)

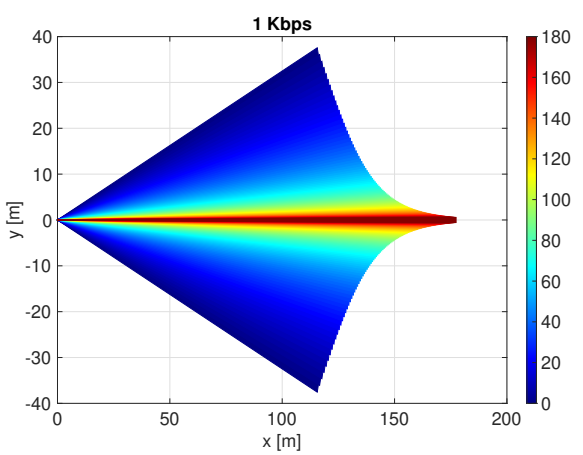

(c) Coverage at 1 Kbps. (BER=FEC)

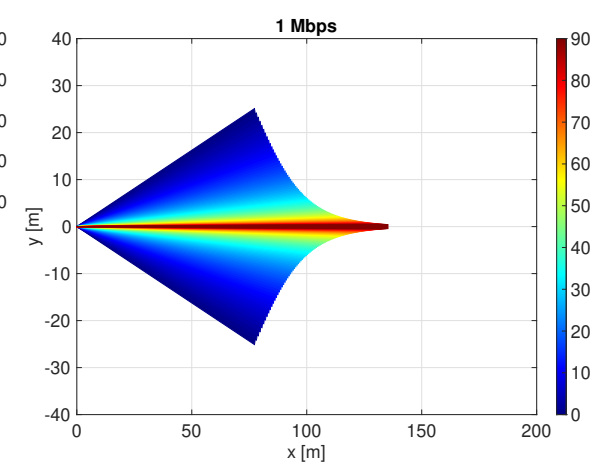

(d) Coverage at $1 \mathrm{Mbps}$. (BER=FEC)

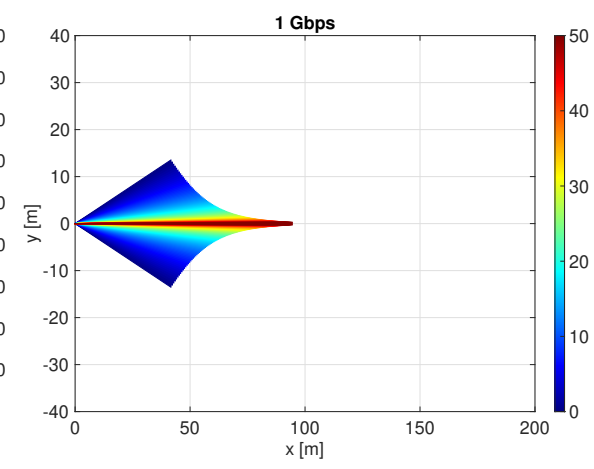

(e) Coverage at 1 Gbps. (BER=FEC)

Fig. 5: Fundamental tradeoffs between key UOWC performance metrics.

explicitly otherwise, we use the parameters listed in Table [I] which is mainly drawn from [6]. Before presenting the numerical results, let us illustrate the operation of SectOR in comparison with a TUR benchmark which is solved Dijkstra's shortest path (DSP). Unlike the SectOR, the DSP calculates the shortest path based on the entire network's available global topology information. Therefore, the DSP sets the divergence angle at its minimum to reach the maximum range with low error performance.

\section{A. Fundamental Tradeoffs among UOWC Metrics}

In order to distinguish SectOR from other geographical routing and OR schemes, it is important to emphasize fundamental trade-offs between key UOWC performance metrics. By using (6) and (9), the data rate and BER are illustrated with respect to the increasing distance between the transceivers in Fig. 5a where BER and data rate are fixed to FEC threshold and 1 Mbps, respectively. It is obvious that they are coupled with the communication range and change oppositely as the distance increases. Fig. 5a also shows that data rate and BER performs differently at different divergence angles (i.e., beamwidths). For example, $1 \mathrm{Mbps}$ rate is achievable at ranges $80 \mathrm{~m}$ and $90 \mathrm{~m}$ by setting the divergence angle to $\theta_{\min }$ and $\theta_{\max }$, respectively. Based on (10), Fig. 5b shows how the communication range decreases as divergence angle and data rate increase when BER is set to FEC. An important conclusion drawn from the $1 \mathrm{Kbps}$ curve in Fig. $5 \mathrm{~b}$ is that UOWC performs not much better than acoustic systems, which can already achieve several Kbps over several hundred meters.

In Fig. 5c 5e, we present the coverage region of a transmitter located at the origin for data rates of $1 \mathrm{Kbps}, 1 \mathrm{Mbps}$, and $1 \mathrm{Gbps}$, respectively. The colorful optical coverage area is obtained by combining sector shapes, which are obtained by changing the divergence angle from $1 \mathrm{mrad}$ to $0.1 \mathrm{rad}$, and the color bar represents the radius of the coverage region at a given beamwidth, i.e., communication range. It can be seen that the coverage region expands as the data requirement is relaxed. It can be deduced from Fig. 5 that UOWC range and coverage is a subjective metric that closely depends on hardware parameters as well as required QoS levels. By comparing Fig. 4 and Fig. 5c 5e, one can also conclude that SectOR takes real-life tradeoffs during the candidate filtering and selection mechanisms, which are indeed crucial to reap the full benefits of the OR concept.

\section{B. Visual Implementation of the SectOR}

Before delving into the numerical results, let us provide a better insight into how the SectOR protocol works. In Fig. 


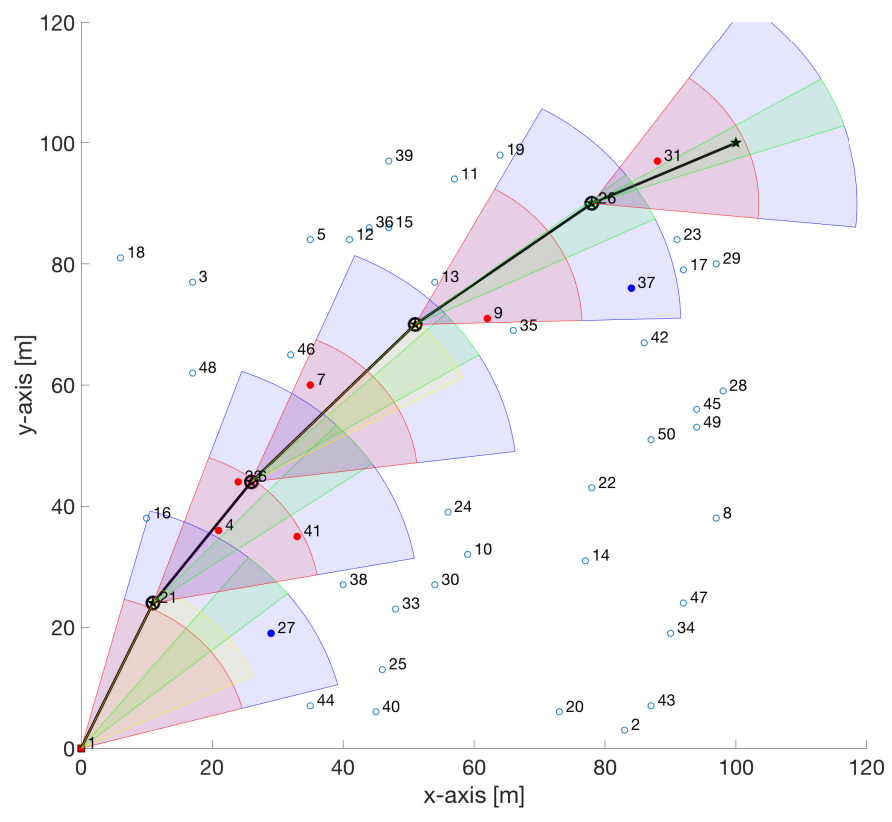

(a)

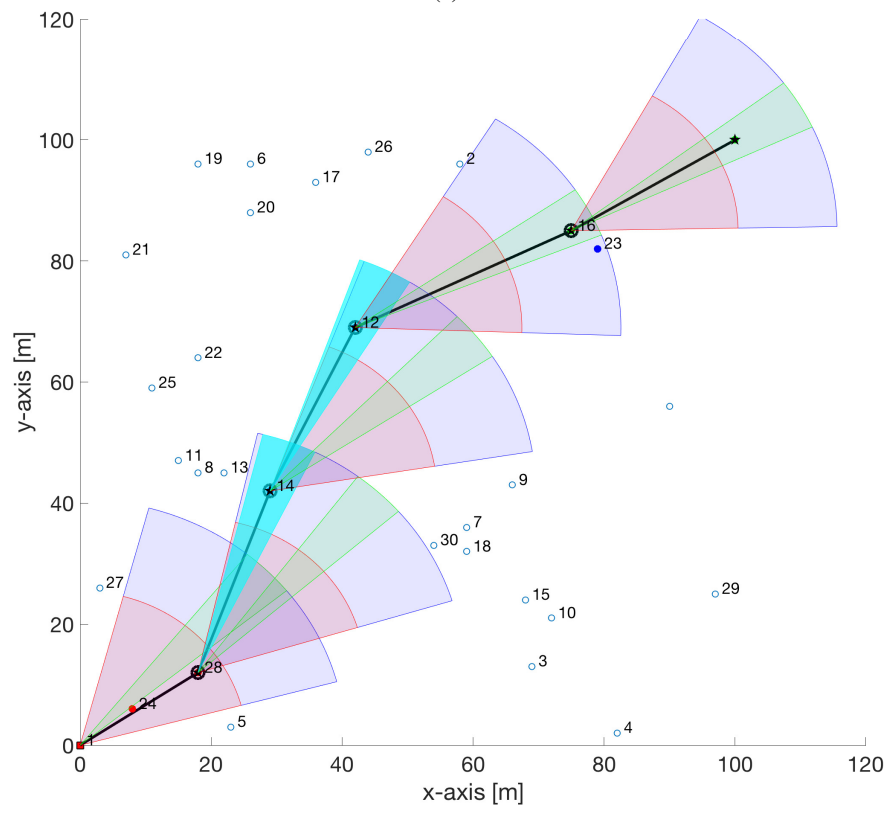

(c)

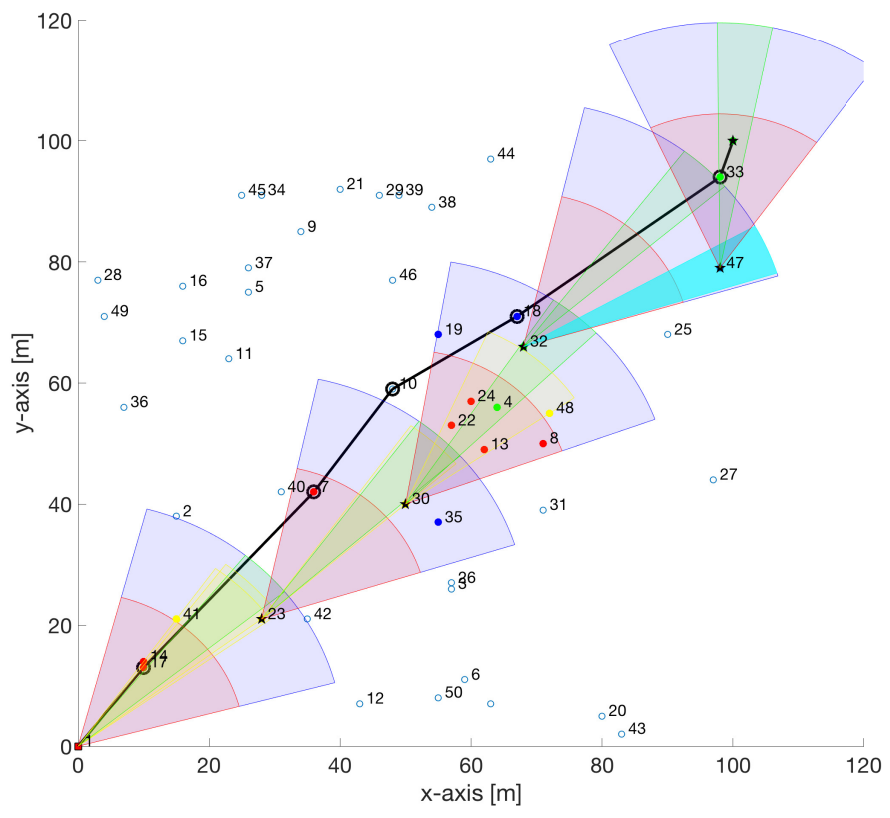

(b)

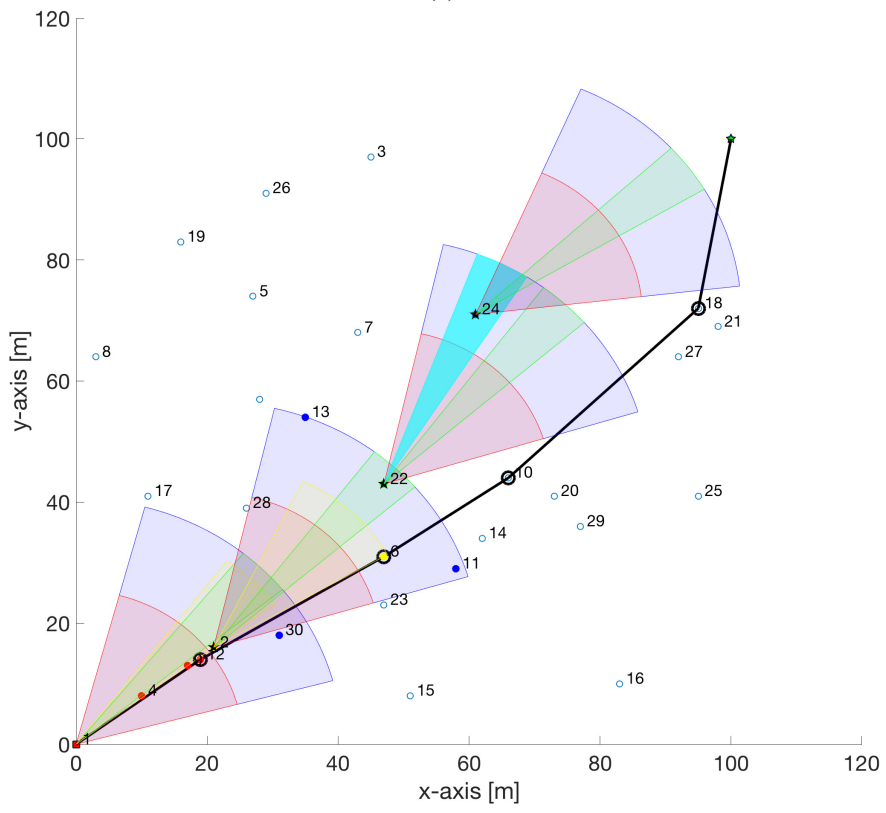

(d)

Fig. 6: Illustration of SectOR in comparison with TUR.

6. solid black lines represent the route calculated by a DSPbased TUR. Blue, red, and green colored sectors correspond to the search space, maximum divergence angle with the minimum range, and minimum divergence angle with the maximum range, respectively. Yellow-colored sectors depict the candidate sets obtained by the DP metric-based candidate selection. Fig. 6a shows an instance where the DP metric yields the same path as the TUR benchmark. Fig. 6b is a clear example of how the SectOR can leverage the proposed candidate selection method to find out potential CSs to reach the destination. Notice that the fourth hop starting from node 32 cannot find any feasible CS if the pointing vector is aligned to the sink node. Therefore, node 32 points the transceiver toward the only node (node 47) that lies within the SS, which is shown by the cyan-colored sector. By doing so, the SectOR was able to reach the sink node via node 47 . A better example of this case is shown in Fig. 6c where the second and third hop is handled by nodes 14 and 12 as there are no nodes within the feasible region of coverage. These alterations are possible since the SectOR is developed to manipulate the physical layer parameters to mitigate the limited range and directivity of the UOWC. Finally, Fig. 6d demonstrates the negative impact of the lack of global topology information on the routing performance. Even though the path is routed over 


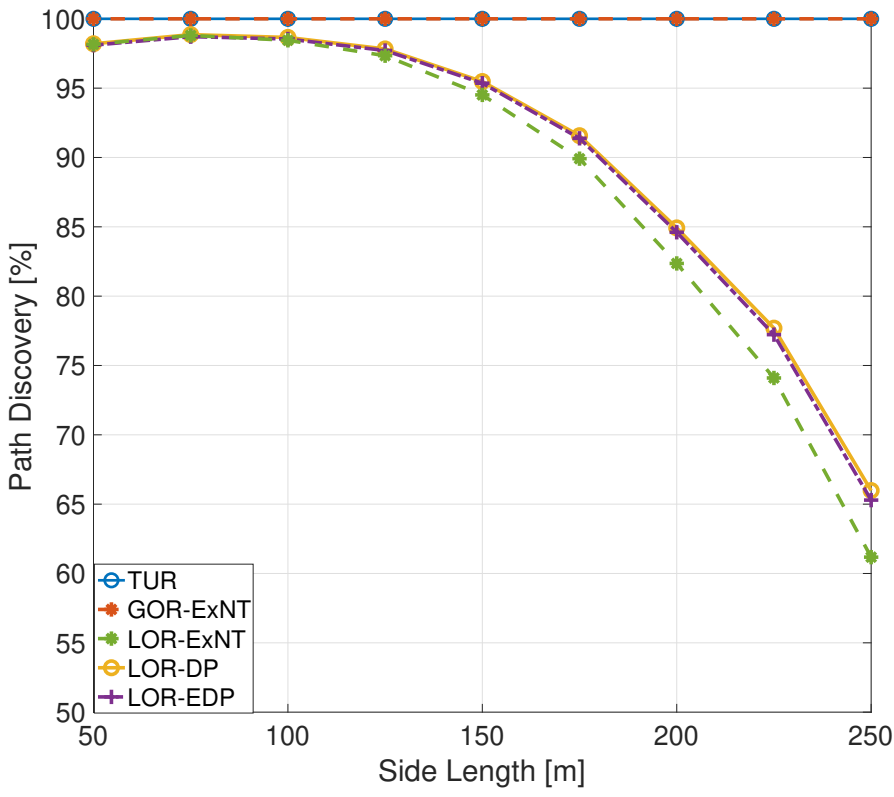

Fig. 7: Percentage of path discovery vs. side length (density).

node 24 by changing the pointing vector node 22 , the local SectOR was not able to reach the destination.

\section{Numerical Analysis}

In the remainder, we present key performance metrics for various network side lengths, i.e., network size. Since we keep the number of nodes constant, increasing side lengths also account for decreasing the node density. In Fig. 7 .Fig. 10 , the EEM and LLM are represented by LOR-ExNT and GORExNT. This is mainly because both local and global energy and delay related metrics are determined by the ExNT metric, which follows from $(25) /(29)$ and $(32) /(36)$, respectively. Fig. 7 demonstrates the impacts of node density on the probability of finding a path from source to destination. Since TUR and GOR-EXNT have a global network view, they could always find a feasible path. On the contrary, LOR methods have a lower probability of path discovery as the node density decreases. In particular, LOR-DP and LOR-EDP perform slightly better than LOR-ExNT because they try to reach the destination in a lower number of hops, which naturally helps them find a feasible path. On the contrary, LOR-ExNT fails to reach the destination while following paths with fewer ExNT.

Fig. 8 demonstrates the fact that E2E-PDR reduces with increasing network sparsity. The first thing to observe is that TUR and LOR-DP perform worse than others, mainly because they are agnostic to the underwater channel hostility and merely consider the distance as their performance metric. By considering the channel condition in tandem with the distance, LOR-EDP delivers much better performance than its counterpart LOR-DP. On the other hand, LOR-ExNT delivers a higher PDR as the ExNT metric is inversely proportional to the PDR. Lastly, GOR-ExNT is the best as it has the global network view. Fig. 9 visualizes the fact that E2E-ExNT increases with decreasing network density. Again, TUR and LOR-DP deliver a poor performance as they only account

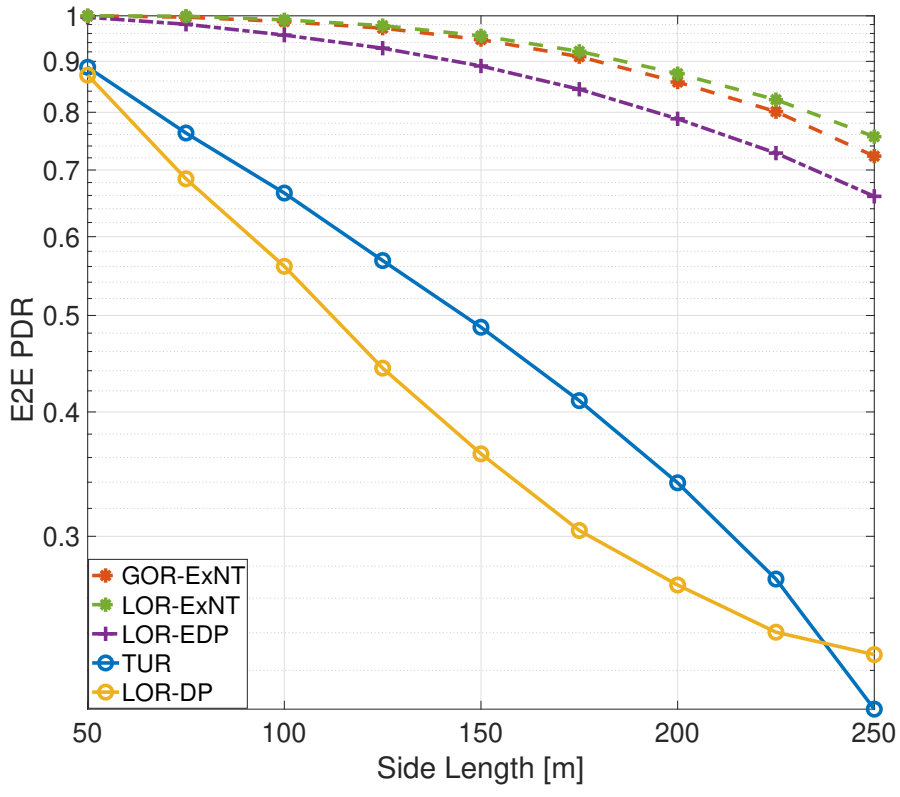

Fig. 8: E2E-PDR vs. side length (density).

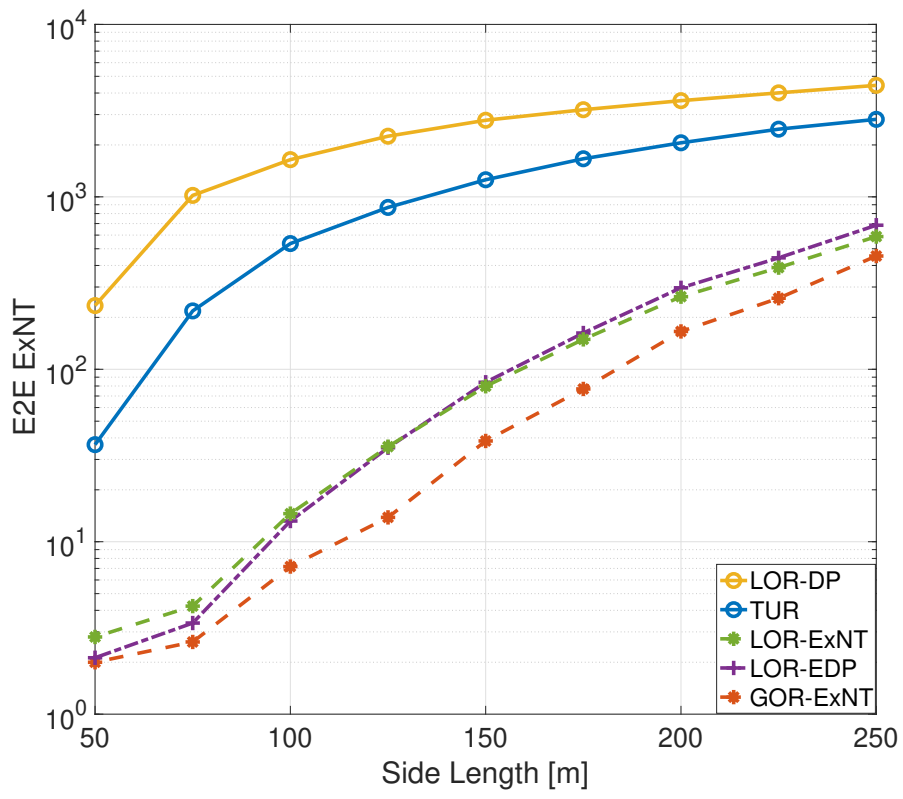

Fig. 9: E2E-ExNT vs. side length (density).

for the distance. On the contrary, LOR-EDP and LOR-EDP improve the E2E-ExNT, which the GOR-ExNT beats at the cost of a higher computational complexity and communication overhead.

Fig. 10 compares the traveled total distance by different schemes. As expected, TUR gives the shortest distance as the DSP is optimal. TUR is followed by LOR-DP and then LOREDP as they account for distance progress. However, LORExNT performs worst as they follow a longer path with a higher number of hops for a minimum ExNT. Although GOREXNT is the worst case under higher densities, it reaches a performance level closer to LOR-DP and LOR-EDP.

Fig. 11 and Fig. 12 show the E2E energy consumption and delay versus varying network size. For the sake of the shortest 


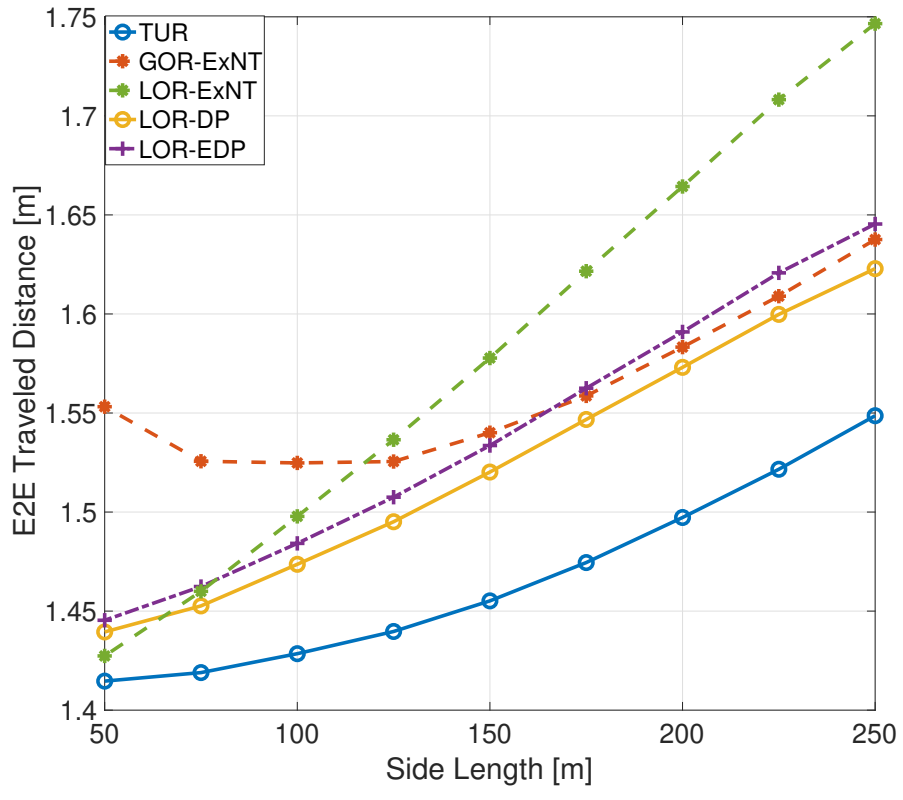

Fig. 10: E2E-distance vs. side length (density).

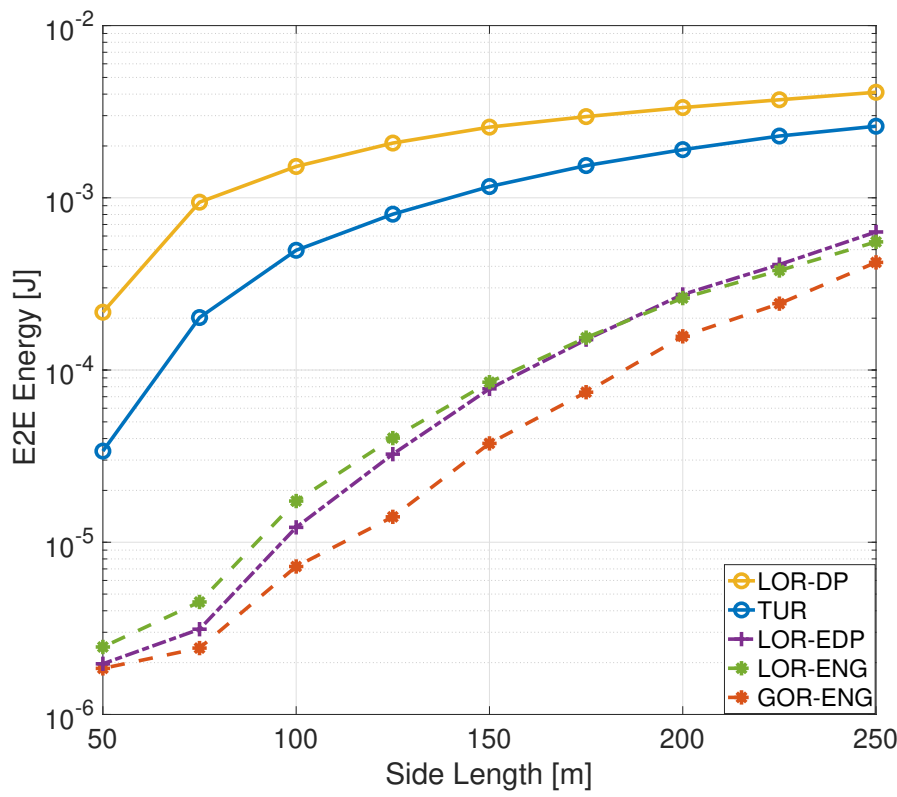

Fig. 11: E2E-Energy vs. side length (density).

path, The LOR-DP yields the highest energy consumption and delays. The TUR provides the second worst-case performance. LOR-EDP and LOR-ExNT improve the energy and delay performance significantly, which is again beaten by the GORENG and GOR-DEL, respectively.

\section{CONCLUSIONS}

In this paper, we developed a sector-based opportunistic routing protocol for UOANs. OR is especially suitable for UOWCs as hostile aquatic channel impairments can disrupt the established link connectivity. By leveraging the broadcast nature of the communication, backing up the broken link connectivity by engaging other users who also received the lost packet may improve the system performance in a significant

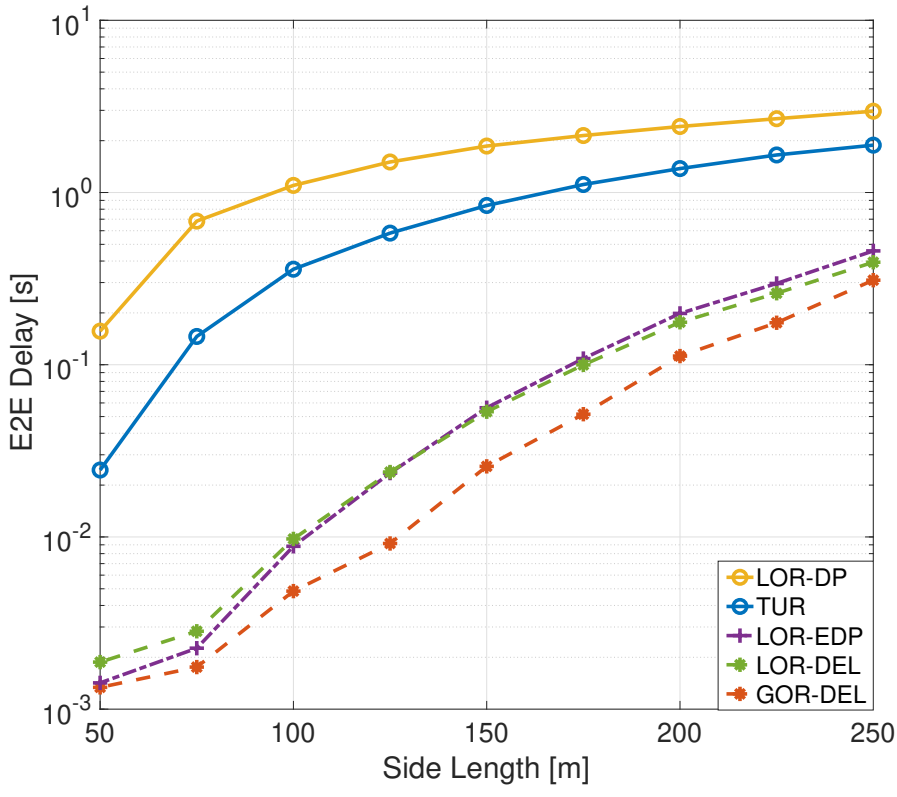

Fig. 12: E2E-Delay vs. side length (density).

extend. The proposed SectOR protocol exploits the sectorshaped coverage region of the light source and finds the path exploiting the local or global topology information in a distributed manner. Numerical results show that it can reach the performance level of a unicast optimal routing especially for high node density levels.

\section{REFERENCES}

[1] A. Celik, N. Saeed, B. Shihada, T. Y. Al-Naffouri, and M.-S. Alouini, "SectOR: Sector-based opportunistic routing protocol for underwater optical wireless networks," in IEEE Wireless Commun. Netw. Conf. (WCNC), Apr. 2019, pp. 1-6.

[2] N. Saeed, A. Celik, T. Y. Al-Naffouri, and M.-S. Alouini, "Underwater optical wireless communications, networking, and localization: A survey," Ad Hoc Networks, vol. 94, p. 101935, 2019.

[3] A. Celik, N. Saeed, B. Shihada, T. Y. Al-Naffouri, and M.-S. Alouini, "A software-defined opto-acoustic network architecture for internet of underwater things," IEEE Commun. Mag., vol. 58, no. 4, pp. 88-94, 2020.

[4] M. Erol-Kantarci, H. T. Mouftah, and S. Oktug, "A survey of architectures and localization techniques for underwater acoustic sensor networks," IEEE Communications Surveys Tutorials, vol. 13, no. 3, pp. 487-502, Third 2011.

[5] A. Celik, N. Saeed, T. Y. Al-Naffouri, and M.-S. Alouini, "Modeling and performance analysis of multihop underwater optical wireless sensor networks," in IEEE Wireless Commun. Netw. Conf. (WCNC), Apr. 2018, pp. 1-6.

[6] A. Celik, N. Saeed, T. Y. Al-Naffouri, and M.-S. Alouini, "End-to-end performance analysis of underwater optical wireless relaying and routing techniques under location uncertainty," IEEE Trans. Wireless Commun. 2019.

[7] LUMA-100 Ultra-efficient wireless optical node, Hydromea. [Online]. Available: https://www.hydromea.com/wp-content/uploads/ 2020/08/Hydromea_LUMA_100_datasheet.pdf

[8] LUMA-250LP Ultra-efficient wireless optical node, Hydromea. [Online]. Available: https://www.hydromea.com/wp-content/uploads/ 2020/08/Hydromea_LUMA_250LP_datasheet.pdf

[9] LUMA-500ER Long range optical modem, Hydromea. [Online]. Available: https://www.hydromea.com/wp-content/uploads/2020/ 08/Hydromea_LUMA_500ER_datasheet.pdf

[10] J. Wang, C. Lu, S. Li, and Z. Xu, "100 m/500 mbps underwater optical wireless communication using an nrz-ook modulated $520 \mathrm{~nm}$ laser diode," Opt. Express, vol. 27, no. 9, pp. 12 171-12 181, Apr 2019. 
[11] N. Farr, A. Bowen, J. Ware, C. Pontbriand, and M. Tivey, "An integrated, underwater optical /acoustic communications system," in OCEANS'10 IEEE SYDNEY, 2010, pp. 1-6.

[12] BlueComm 200 - Optical Wireless Underwater Video and Vehicle Control, Sonardyne. [Online]. Available: https://www.sonardyne.com/ app/uploads/2016/06/Sonardyne_8361_BlueComm_200.pdf

[13] I. F. Akyildiz, P. Wang, and S.-C. Lin, "Softwater: Software-defined networking for next-generation underwater communication systems," Ad Hoc Networks, vol. 46, pp. 1 - 11, 2016.

[14] N. Saeed, A. Celik, T. Y. Al-Naffouri, and M.-S. Alouini, "Connectivity analysis of underwater optical wireless sensor networks: A graph theoretic approach," in IEEE Int. Conf. Commun. Workshops (ICC Workshops), May 2018, pp. 1-6.

[15] M. V. Jamali, F. Akhoundi, and J. A. Salehi, "Performance characterization of relay-assisted wireless optical CDMA networks in turbulent underwater channel," IEEE Trans. Wireless Commun., vol. 15, no. 6, pp. 4104-4116, June 2016.

[16] M. V. Jamali, A. Chizari, and J. A. Salehi, "Performance analysis of multi-hop underwater wireless optical communication systems," IEEE Photon. Technol. Lett., vol. 29, no. 5, pp. 462-465, March 2017.

[17] R. Diamant, P. Casari, F. Campagnaro, and M. Zorzi, "Routing in multimodal underwater networks: A throughput-optimal approach," in 2017 IEEE Conference on Computer Communications Workshops (INFOCOM WKSHPS), 2017, pp. 205-210.

[18] N. Chakchouk, "A survey on opportunistic routing in wireless communication networks," IEEE Communications Surveys Tutorials, vol. 17, no. 4, pp. 2214-2241, Fourthquarter 2015.

[19] V. G. Menon and P. M. J. Prathap, "Comparative analysis of opportunistic routing protocols for underwater acoustic sensor networks," in Intl. Conf. Emerging Techno. Trends (ICETT), Oct 2016, pp. 1-5.

[20] A. Darehshoorzadeh and A. Boukerche, "Underwater sensor networks: a new challenge for opportunistic routing protocols," IEEE Commun. Mag., vol. 53, no. 11, pp. 98-107, Nov. 2015.

[21] R. W. L. Coutinho, A. Boukerche, L. F. M. Vieira, and A. A. F. Loureiro, "Geographic and opportunistic routing for underwater sensor networks," IEEE Trans. Computers, vol. 65, no. 2, pp. 548-561, Feb. 2016.

[22] —, "GEDAR: Geographic and opportunistic routing protocol with depth adjustment for mobile underwater sensor networks," in IEEE Intl. Conf. Commun. (ICC), Jun. 2014, pp. 251-256.

[23] P. LoPresti, H. Refai, J. Sluss, and I. Varela-Cuadrado, "Adaptive divergence and power for improving connectivity in free-space optical mobile networks," Appl. Opt., vol. 45, no. 25, pp. 6591-6597, Sep 2006.

[24] A. A. Aleksandrov, A. V. Kudryashov, A. L. Rukosuev, T. Y. Cherezova, and Y. V. Sheldakova, "An adaptive optical system for controlling laser radiation," J. Opt. Technol., vol. 74, no. 8, pp. 550-554, Aug 2007.

[25] M. Rahm, D. A. Roberts, J. B. Pendry, and D. R. Smith, "Transformation-optical design of adaptive beam bends and beam expanders," Opt. Express, vol. 16, no. 15, pp. 11 555-11 567, Jul 2008.

[26] P. Brandl, S. Schidl, A. Polzer, W. Gaberl, and H. Zimmermann, "Optical wireless communication with adaptive focus and mems-based beam steering," IEEE Photonics Technology Letters, vol. 25, no. 15, 2013.

[27] V. V. Mai and H. Kim, "Adaptive beam control techniques for airborne free-space optical communication systems," Appl. Opt., vol. 57, no. 26, pp. 7462-7471, Sep 2018.

[28] P. Xie, J.-H. Cui, and L. Lao, "VBF: Vector-based forwarding protocol for underwater sensor networks," in NETWORKING 2006. Networking Technologies, Services, and Protocols; Performance of Computer and Communication Networks; Mobile and Wireless Communications Systems, F. Boavida, T. Plagemann, B. Stiller, C. Westphal, and E. Monteiro, Eds. Berlin, Heidelberg: Springer Berlin Heidelberg, 2006, pp. 12161221.

[29] Daeyoup Hwang and Dongkyun Kim, "DFR: Directional flooding-based routing protocol for underwater sensor networks," in OCEANS 2008, Sep. 2008, pp. 1-7.

[30] J. M. Jornet, M. Stojanovic, and M. Zorzi, "Focused beam routing protocol for underwater acoustic networks," in Proceedings of the Third ACM International Workshop on Underwater Networks, ser. WuWNeT '08. New York, NY, USA: ACM, 2008, pp. 75-82.

[31] N. Saeed, A. Celik, M.-S. Alouini, and T. Y. Al-Naffouri, "Energy harvesting hybrid acoustic-optical underwater wireless sensor networks localization," Sensors, vol. 18, no. 1, 2017.

[32] P. LoPresti, H. Refai, J. Sluss, and I. Varela-Cuadrado, "Adaptive divergence and power for improving connectivity in free-space optical mobile networks," Appl. Opt., vol. 45, no. 25, pp. 6591-6597, Sep. 2006.

[33] N. Saeed, A. Celik, T. Y. Al-Naffouri, and M.-S. Alouini, "Localization of energy harvesting empowered underwater optical wireless sensor networks," IEEE Trans. Wireless Commun., vol. 18, no. 5, pp. 26522663, May 2019.

[34] — - "Performance analysis of connectivity and localization in multihop underwater optical wireless sensor networks," IEEE Trans. Mobile Comput., vol. 18, no. 11, pp. 2604-2615, Nov 2019.

[35] N. Saeed, A. Celik, M.-S. Alouini, and T. Y. Al-Naffouri, "Analysis of $3 \mathrm{~d}$ localization in underwater optical wireless networks with uncertain anchor positions," Science China Information Sciences, vol. 63, no. 10, pp. $1-8,2020$.

[36] J. Poliak, P. Pezzei, E. Leitgeb, and O. Wilfert, "Link budget for highspeed short-distance wireless optical link," in 2012 8th International Symposium on Communication Systems, Networks Digital Signal Processing (CSNDSP), Jul. 2012, pp. 1-6.

[37] M. Elamassie, F. Miramirkhani, and M. Uysal, "Performance characterization of underwater visible light communication," IEEE Trans. Commun., pp. 1-1, 2018.

[38] S. Arnon, "Underwater optical wireless communication network," Optical Engineering, vol. 49, pp. 49 - 49 - 6, 2010.

[39] M. Zorzi and R. R. Rao, "Geographic random forwarding (GeRaF) for ad hoc and sensor networks: multihop performance," IEEE Trans. Mobile Comput., vol. 2, no. 4, pp. 337-348, Oct. 2003.

[40] A. Darehshoorzadeh and L. Cerdà-Alabern, "Distance progress based opportunistic routing for wireless mesh networks," in 2012 8th International Wireless Communications and Mobile Computing Conference (IWCMC), Aug 2012, pp. 179-184.

[41] S. Biswas and R. Morris, "Opportunistic routing in multi-hop wireless networks," SIGCOMM Comput. Commun. Rev., vol. 34, no. 1, pp. 6974, Jan. 2004

[42] A. Zubow, M. Kurth, and J.-P. Redlich, "Multi-channel opportunistic routing," in European Wireless Conference (EW), 2007.

[43] Z. Yang, K. Zeng, and W. Lou, "FSA: A fast coordination scheme for opportunistic routing," in IEEE Intl. Conf. Commun. (ICC), Jun 2009, pp. $1-5$.

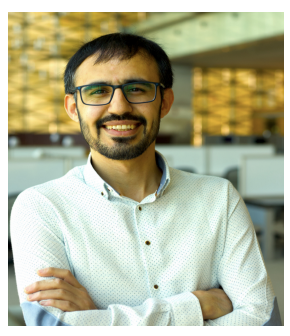

Abdulkadir Celik (Senior Member, IEEE) received the M.S. degree in electrical engineering in 2013 , the M.S. degree in computer engineering in 2015, and the Ph.D. degree in co-majors of electrical engineering and computer engineering in 2016 from Iowa State University, Ames, IA, USA. He was a post-doctoral fellow at King Abdullah University of Science and Technology (KAUST) from 2016 to 2020. Since 2020, he has been a research scientist at the communications and computing systems lab at KAUST. His research interests are in the areas of wireless communication systems and networks.

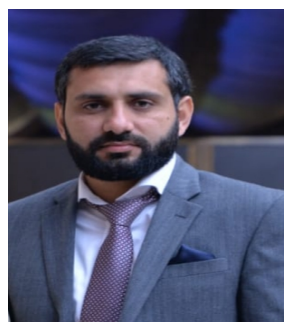

Nasir Saeed (Senior Member, IEEE) received the bachelor's degree in telecommunication from the University of Engineering and Technology, Peshawar, Pakistan, in 2009, the master's degree in satellite navigation from the Polito di Torino, Italy, in 2012, and the Ph.D. degree in electronics and communication engineering from Hanyang University, Seoul, South Korea, in 2015. He was an Assistant Professor with the Department of Electrical Engineering, Gandhara Institute of Science and IT, Peshawar, from August 2015 to September 2016. $\mathrm{He}$ has worked as an Assistant Professor with IQRA National University, Peshawar, from October 2016 to July 2017. From July 2017 to December 2020, he was a Postdoctoral Research Fellow with the Communication Theory Laboratory, King Abdullah University of Science and Technology (KAUST). $\mathrm{He}$ is currently an Associate Professor with the Department of Electrical Engineering, National University of Technology (NUTECH), Islamabad, Pakistan. His current research interests include cognitive radio networks, underwater wireless communications, aerial networks, dimensionality reduction, and localization. 


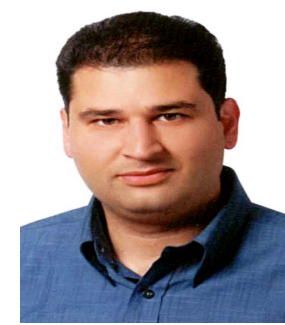

Basem Shihada (Senior Member, IEEE) is an associate \& founding professor in the Computer, Electrical and Mathematical Sciences \& Engineering (CEMSE) Division at King Abdullah University of Science and Technology (KAUST). He obtained his $\mathrm{PhD}$ in Computer Science from University of Waterloo. In 2009, he was appointed as visiting faculty in the Department of Computer Science, Stanford University. In 2012, he was elevated to the rank of Senior Member of IEEE. His current research covers a range of topics in energy and resource allocation in wired and wireless networks, software defined networking, internet of things, data networks, network security, and cloud/fog computing.

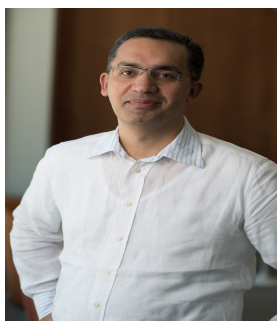

Tareq Y. Al-Naffouri (Senior Member, IEEE) Tareq Al-Naffouri received the B.S. degrees in mathematics and electrical engineering (with first honors) from King Fahd University of Petroleum and Minerals, Dhahran, Saudi Arabia, the M.S. degree in electrical engineering from the Georgia Institute of Technology,Atlanta, in 1998, and the Ph.D. degree in electrical engineering from Stanford University, Stanford, CA, in 2004.

He was a visiting scholar at California Institute of Technology, Pasadena, CA in 2005 and summer 2006. He was a Fulbright scholar at the University of Southern California in 2008. He is currently a Professor at the Electrical Engineering Department, King Abdullah University of Science and Technology (KAUST). His research interests lie in the areas of sparse, adaptive, and statistical signal processing and their applications to wireless communications and localization, machine learning, and network information theory. He has over 300 publications in journal and conference proceedings and 20 issued/pending patents.

Dr. Al-Naffouri is the recipient of the IEEE Education Society Chapter Achievement Award in 2008 and Al-Marai Award for innovative research in communication in 2009. Dr. Al-Naffouri was an Associate Editor of IEEE Transactions on Signal Processing 2013-2018.

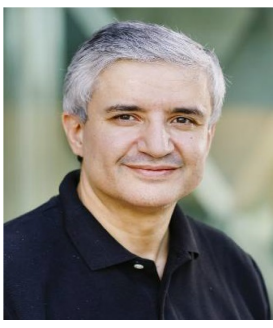

Mohamed-Slim Alouini (Fellow, IEEE) was born in Tunis, Tunisia. He received the Ph.D. degree in Electrical Engineering from the California Institute of Technology (Caltech), Pasadena, CA, USA, in 1998. He served as a faculty member in the University of Minnesota, Minneapolis, MN, USA, then in the Texas A\&M University at Qatar, Education City, Doha, Qatar before joining King Abdullah University of Science and Technology (KAUST), Thuwal, Makkah Province, Saudi Arabia as a Professor of Electrical Engineering in 2009. His current research interests include the modeling, design, and performance analysis of wireless communication systems. 